\title{
Localization of Classical Waves I: Acoustic Waves
}

\author{
Alexander Figotin, ${ }^{1, \star}$ Abel Klein ${ }^{2, \star \star}$ \\ 1 Department of Mathematics, Univessity of North Carolina at Charlotte, Charlotte, NC 28223, \\ USA E-mail: figotin@mosaic uncc edu \\ 2 Department of Mathematics, University of California, Irvine, Irvine, CA 92697-3875, USA \\ E-mail: aklein@math uci edu
}

Received: 22 November 1995/Accepted: 29 February 1996

\begin{abstract}
We consider classical acoustic waves in a medium described by a position dependent mass density $\varrho(x)$. We assume that $\varrho(x)$ is a random perturbation of a periodic function $\varrho_{0}(x)$ and that the periodic acoustic operator $A_{0}=-\nabla \cdot \frac{1}{Q_{0}(x)} \nabla$ has a gap in the spectrum We prove the existence of localized waves, i e., finite energy solutions of the acoustic equations with the property that almost all of the wave's energy remains in a fixed bounded region of space at all times, with probability one Localization of acoustic waves is a consequence of Anderson localization for the self-adjoint operators $A=-\nabla \cdot \frac{1}{\varrho(x)} \nabla$ on $L^{2}\left(\mathbb{R}^{d}\right)$ We prove that, in the random medium described by $\varrho(x)$, the random operator $A$ exhibits Anderson localization inside the gap in the spectrum of $A_{0}$ This is shown even in situations when the gap is totally filled by the spectrum of the random operator, we can prescribe random environments that ensure localization in almost the whole gap.
\end{abstract}

\section{Contents}

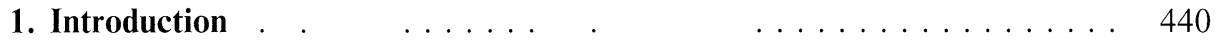

11. Acoustic waves and localization $\quad . \ldots \ldots \ldots 441$

1.2 Statement of results . . . . . . . . . . . . . . 442

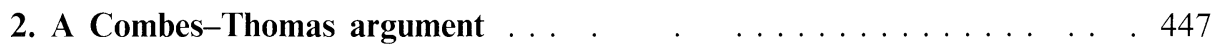

3. Periodic operators and periodic boundary condition . . 449

3.1 Periodic boundary condition .... . . . . . . . 450

32 Spectrum of periodic operators ... . . . . 450

33 A Combas-Thomas argument for the torus . . 455

4. Location of the spectrum of random operators _ . . . . 456

41 Approximation by periodic operators $\ldots \ldots \ldots \ldots 456$

42 Inside the gap . . . . . . . 460

$\star$ This author was supported by the U S Air Force Grant F49620-94-1-0172

$\star \star$ This author was supported in part by the NSF Grants DMS-9208029 and DMS-9500720 


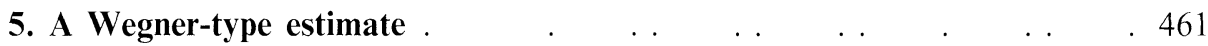

6. Localization . . . $\quad$. $\quad$. 464

61 The basic technical tools . . . . . . . . . . 464

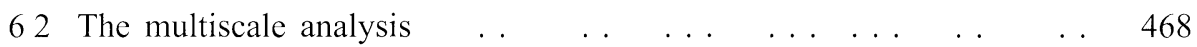

63 Boundary condition. from periodic to Dirichlet . . . . . . . 473

64 Localization in a specific interval . . . . . . . . . . . . . . . 474

65 Localization at the edge $\quad \ldots \quad \ldots \quad \ldots \quad \ldots \quad \ldots \quad \ldots 46$

A. Measurability of operator valued functions . . . . . . . . . . . . 476

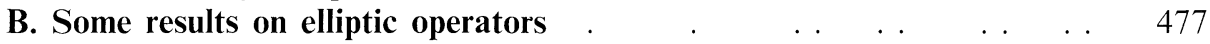

B.1 An interior estimate . . . . . . . . . . . . . . 477

B.2 Generalized eigenfunctions . $\quad \ldots \quad \ldots \quad \ldots \quad \ldots \quad \ldots \quad \ldots \quad \ldots 478$

C. Lemmas on convergence of operators . _ . . . . . . . . . 479

\section{Introduction}

Localization of classical waves, acoustic and electromagnetic, has received much attention in recent years (e g., [An2, J1, J2, DE, Sc, VP, M] and references therein) This phenomenon arises from coherent multiple scattering and interference and occurs when the scale of the coherent multiple scattering reduces to the wavelength itself. Numerous potential applications (e g, [DE, J2, VP]), for instance, the optical transistor, and the fundamental significance of localization of classical waves motivate the interest in this phenomenon

In spite of the clear similarities between localization of quantum-mechanical electrons (studied by Anderson [Anl] for the tight binding model), and localization of classical waves, there are some important differences In particular, classical waves are harder to localize [J2]. A substantial difference is apparent when we multiply the coefficient describing the medium (the position dependent density for acoustic media, the dielectric constant for the dielectric media, the potential for electrons) by a constant for classical waves the spectrum of the relevant operators will be just rescaled, in contrast to the case of Schrödinger operators. Another significant difference is that a local change in a homogeneous medium cannot create localized eigenfunctions for classical waves operators, but it can certainly create localized states for Schrödinger operators For classical waves the bottom of the spectrum is always at 0 and clearly does not depend on the medium; for Schrödinger operators the bottom of the spectrum is movable so we may expect the rise of localized states for appropriate potentials

Thus, in order for localization of classical waves to occur we have to play with the space distribution of inhomogeneities S John [J2] writes Photon localization arises here not as the by-product of high degree of uncontrolled disorder, but rather as a result of a subtle interplay between order and disorder The true criterion for localization, in fact, depends strongly on the underlying static structure factor of the medium This suggests that the localization of classical waves can be achieved only in appropriately prepared random environments

To create an environment which would favor localization one considers first a perfectly periodic medium It is well known that the spectrum associated with a periodic medium has band gap structure and that the most significant manifestation of coherent multiple scattering is the rise of a gap in the spectrum If such 
a periodic medium with a gap in the spectrum is slightly randomized, eigenvalues with exponentially localized eigenfunctions should arise in the gap. If the disorder is increased further within some limits the localized states can fill the gap completely. This is exactly the medium in which we study acoustic and electromagnetic waves Acoustic waves are treated in this paper, electromagnetic waves will be discussed in a sequel [FK3] Localized classical waves created by local defects are studied in [FK4] We assume that the underlying periodic medium has a gap in the spectrum The existence of periodic media exhibiting gaps in the spectrum is proved for acoustic and 2D-periodic dielectric structures [FKu1, FKu2] We will slightly randomize such periodic media with a gap in the spectrum and show that, under pretty reasonable hypotheses, Anderson localization occurs in a vicinity of the edges of the gap.

We previously considered these questions and media in a lattice approximation, both for classical waves [FK2] and for Schrödinger operators [FK1] The strategy of this paper is the same one we used in [FK2], the main differences are of technical nature and due to working on the continuum instead of the lattice

11 Acoustic waves and localization In an inhomogeneous medium the basic linear acoustic equations are (e.g., $[\mathrm{MI}])$

$$
\kappa \frac{\partial p}{\partial t}=-\nabla \cdot \mathbf{u}, \quad \varrho \frac{\partial \mathbf{u}}{\partial t}=-\nabla p,
$$

where $p=p(x, t)$ is the pressure, $\mathbf{u}=\mathbf{u}(x, t)$ is the velocity, $\kappa=\kappa(x)$ is the compressibility, and $\varrho=\varrho(x)$ is the mass density, so the pressure satisfies the second order partial differential equation

$$
\kappa \frac{\partial^{2} p}{\partial t^{2}}=\nabla \cdot \frac{1}{\varrho} \nabla p
$$

The energy density $\mathscr{E}(x, t)$ and the (conserved) energy $\mathscr{E}$ are given by

$$
\mathscr{E}(x, t)=\frac{1}{2}\left[\varrho(x)|u(x, t)|^{2}+\kappa(x)|p(x, t)|^{2}\right], \quad \mathscr{E}=\int \mathscr{E}(x, t) d x
$$

It is convenient to introduce the momentum potential $\Psi=\Psi(x, t)$ by $\varrho u=-\nabla \Psi$, and rewrite (1) as

$$
\kappa \frac{\partial p}{\partial t}=\nabla \cdot \frac{1}{\varrho} \nabla \Psi, \quad \frac{\partial \Psi}{\partial t}=\nabla p .
$$

It follows that $\Psi$ obeys the same second order partial differential equation (2) as the pressure $p$, i.e,

$$
\kappa \frac{\partial^{2} \Psi}{\partial t^{2}}=\nabla \cdot \frac{1}{\varrho} \nabla \Psi
$$

and the energy density can be rewritten as

$$
\mathscr{E}(x, t)=\frac{1}{2}\left[\varrho(x)|\nabla \Psi(x, t)|^{2}+\kappa(x)\left|\frac{\partial \Psi}{\partial t}(x, t)\right|^{2}\right] .
$$

Motivated by (2), (4) and (5), we set $A=-\nabla \cdot \frac{1}{Q} \nabla$ It follows from (4) that $\Psi=-A^{-1} \kappa \frac{\partial p}{\partial t}$, so we can recover the velocity $\mathbf{u}$ from the pressure $p$ by 
$\mathbf{u}=\frac{1}{\varrho} \nabla A^{-1} \kappa \frac{i p}{\hat{c} t}$, so the energy density can also be written in terms of the pressure as

$$
\mathscr{E}(x, t)=\frac{1}{2}\left[\frac{1}{\varrho(x)}\left|\nabla\left(A^{-1} \kappa \frac{\partial p}{\partial t}\right)(x, t)\right|^{2}+\kappa(x)|p(x, t)|^{2}\right] .
$$

In this article we work with a position dependent mass density $\varrho(x)$ and constant compressibility $\kappa$ (we set $\kappa=1$ ). We will define $A$ as a nonnegative self-adjoint operator on $L^{2}\left(\mathbb{R}^{d}\right)$ Finite energy solutions for Eqs (2) and (5) are given by (e g, [RS3, Sect IX 10])

$$
\varphi(t)=\cos \left(t A^{\frac{1}{2}}\right) \varphi_{0}+A^{-\frac{1}{2}} \sin \left(t A^{\frac{1}{2}}\right) \dot{\varphi}_{0},
$$

where the Cauchy data $\left(\varphi_{0}, \dot{\varphi}_{0}\right)$ can be taken in $L^{2}\left(\mathbb{R}^{d}\right) \times \mathscr{Z}\left(A^{-\frac{1}{2}}\right)$ for the pressure $p$, and in $\mathscr{T}\left(A^{\frac{1}{2}}\right) \times L^{2}\left(\mathbb{R}^{d}\right)$ for the potential $\Psi$

A localized acoustic wave should be a finite energy solution of (1) with the property that almost all of the wave's energy remains in a fixed bounded region of space at all times, e.g,

$$
\lim _{R \rightarrow \infty} \inf _{t} \frac{1}{\mathscr{E}} \int_{|x| \leqq R} \mathscr{E}(x, t) d x=1
$$

Our strategy for proving the existence localized waves is the following we first prove that the operator A has pure point spectrum in some closed interval $I \subset(0, \infty)$, with all the corresponding eigenfunctions being exponentially decaying For this operator it will follow that the gradient of an exponentially decaying eigenfunction has exponentially decaying local $L^{2}$-norms, so the corresponding energy' densities (given by either (6) or (7)) also have exponentially decaying local $L^{2}$-norms, uniformly in the time $t$ If $\chi_{I}(A)$ is the corresponding spectral projection, then any solution of either (2) or (5) given by (8), with Cauchy data in the range of $\chi_{I}(A)$, satisfies $(9)$

Localization of acoustic waves is thus a consequence of Anderson localization for operators $A=-\nabla \cdot \frac{1}{Q} \nabla$ on $L^{2}\left(\mathbb{R}^{d}\right), i$ e., the existence of closed intervals where these operators have pure point spectrum with exponentially decaying eigenfunctions

Similarly, the localization of electromagnetic waves is a consequence of Anderson localization for operators $M=\nabla \times \frac{1}{\varepsilon} \nabla \times$ on $L^{2}\left(\mathbb{R}^{3}, \mathbb{C}^{3}\right)$, where $\varepsilon=\varepsilon(x)$ is the position dependent dielectric constant. Localization of electromagnetic waves will be studied in a sequel to this article [FK3].

12 Statement of results We study acoustic waves which are described by the following formally self-adjoint operator on $L^{2}\left(\mathbb{R}^{d}\right)$ :

$$
A=A(\varrho)=-\nabla \cdot \frac{1}{\varrho(x)} \nabla=-\sum_{j=1}^{d} \partial_{j} \frac{1}{\varrho(x)} \partial_{J},
$$

where we always assume that $\varrho(x)$ is a measurable real valued function satisfying

$$
0<\varrho_{-} \leqq \varrho(x) \leqq \varrho_{+}<\infty \text { a e for some constants } \varrho_{-} \text {and } \varrho_{+}
$$

Such general conditions on $Q(x)$, particularly the lack of smoothness, are required on physical grounds. In practice only a few materials are used in the fabrication 
of periodic and disordered media, in which case $\varrho(x)$ takes just a finite number of values, so $Q(x)$ is piecewise constant, hence discontinuous. The abrupt changes in the medium produce discontinuities in $\varrho(x)$, which favor and enhance multiscattering and, hence, localization

$A$ is rigorously defined as the unique nonnegative self-adjoint operator on $L^{2}\left(\mathbb{R}^{d}\right)$ uniquely defined by the quadratic form given as the closure of the nonnegative densely defined quadratic form

$$
\mathscr{A}(\psi)=\left\langle\nabla \psi, \frac{1}{\varrho(x)} \nabla \psi\right\rangle \equiv \sum_{j=1}^{d}\left\langle\partial_{j} \psi, \frac{1}{\varrho(x)} \partial_{,} \psi\right\rangle, \quad \text { with } \psi \in C_{0}^{1}\left(\mathbb{R}^{d}\right) .
$$

In this article we consider acoustic waves in random media obtained by random perturbations of a periodic medium. The properties of the medium are described by the position dependent quantity $\varrho(x)$, which we will always take to satisfy the following assumptions

Assumption 1 (The Random Media). $\varrho_{g}(x)=\varrho_{g,(1)}(x)$ is a random function of the form

$$
\varrho_{g,(1)}(x)=\varrho_{0}(x) \gamma_{g, \omega}(x), \quad \text { with } \gamma_{g, \omega}(x)=1+g \sum_{l \in \mathbb{Z}^{d}} \omega_{i} u_{i}(x),
$$

where

(i) $\varrho_{0}(x)$ is a measurable real valued function which is q-periodic for some $q \in \mathbb{N}$, $i e, \varrho_{0}(x)=\varrho_{0}(x+q i)$ for all $x \in \mathbb{R}$ and $i \in \mathbb{Z}^{d}$, with

$$
0<\varrho_{0,-} \leqq \varrho_{0}(x) \leqq \varrho_{0,+}<\infty \quad \text { for a e } x \in \mathbb{R}^{d}
$$

for some constants $\varrho_{0,-}$ and $\varrho_{0 .+}$

(ii) $u_{l}(x)=u(x-i)$ for each $i \in \mathbb{Z}^{d}$, u being a nonnegative measurable real valued function with compact support, say $u(x)=0$ if $\|x\|_{\infty} \leqq r_{u}$ for some $r_{u}<$ $\infty$, such that

$$
0<U_{-} \leqq U(x) \equiv \sum_{i \in \mathbb{Z}^{d}} u_{l}(x) \leqq U_{+}<\infty \quad \text { for a e } x \in \mathbb{R}^{d},
$$

for some constants $U_{-}$and $U_{+}$

(iii) $\omega=\left\{\omega_{l}, i \in \mathbb{Z}^{d}\right\}$ is a family of independent, identically distributed random variables taking values in the interval $[-1,1]$, whose common probability distribution $\mu$ has a bounded density $\rho>0$ a e in $[-1,1]$

(iv) $g$, satisfying $0 \leqq g<\frac{1}{U_{i}}$, is the disorder parameter

For acoustic waves $\varrho_{y, \omega}(x)$ is the random position dependent mass density of the medium Notice that Assumption 1 implies that each $\varrho_{g,(1)}$ satisfies (11) with

$$
\varrho_{ \pm}=\varrho_{g, \pm}=\varrho_{0, \pm}\left(1 \pm g U_{+}\right) \text {. }
$$

For later use we set

$$
\delta_{ \pm}(g)=\frac{U_{ \pm}}{1 \mp g U_{+}} \quad \text { with } 0 \leqq g<\frac{1}{U_{+}}
$$

The periodic operator associated with the coefficient $\varrho_{0}(x)$ is denoted by $A_{0}, \mathrm{i}$ e , $A_{0}=A\left(\varrho_{0}\right)$ We will study the random acoustic operators (see Appendix A)

$$
A_{g}=A_{g,(1)}=A\left(\varrho_{g,(1)}\right)
$$


It follows from ergodicity (measurability follows from Theorem 38 below) that there exists a nonrandom set $\Sigma_{y}$, such that $\sigma\left(A_{g .(1)}\right)=\Sigma_{g}$ with probability one, where $\sigma(A)$ denotes the spectrum of the operator $A$ In addition, the decomposition of $\sigma\left(A_{y_{\text {, }}(1)}\right)$ into pure point spectrum, absolutely continuous spectrum and singular continuous spectrum is also independent of the choice of $\omega$ with probability one [KM1, PF]

In this article we are interested in the phenomenon of localization According to the philosophy of Anderson localization we will assume that the operator $A_{0}$ has at least one gap in the spectrum

Assumption 2 (The gap in the spectrum). There is a gap in the spectrum of the operator $A_{0}$ More precisely, there exist $0 \leqq \hat{a}<a<b<\hat{b}$ such that

$$
\sigma\left(A_{0}\right) \cap[\hat{a}, \hat{b}]=[\hat{a}, a] \cup[b, \hat{b}],
$$

so the interval $(a, b)$ is a gap in $\sigma\left(A_{0}\right)$

The following theorem gives information on the location of $\Sigma_{g}$, the (nonrandom) spectrum of the random acoustic operator $A_{g}$

Theorem 3 (Location of the Spectrum). Let the random operator $A_{g}$ defined by (18) satisfy Assumptions 1 and 2 There exists $g_{0}$, with

$$
\frac{1}{U_{+}}\left(1-\left(\frac{a}{b}\right)^{\frac{1}{2}}\right) \leqq g_{0} \leqq \frac{1}{U_{+}} \min \left\{1,\left(\left(\frac{b}{a}\right)^{\frac{c_{+}}{2 U_{-}}}-1\right)\right\}
$$

and strictly increasing, Lipschitz continuous real valued functions $a(g)$ and $-b(g)$ on the interval $\left[0, \frac{1}{U_{+}}\right)$, with $a(0)=a, b(0)=b$ and $a(g) \leqq b(g)$, such that

$$
\Sigma_{g} \cap[\hat{a}, \hat{b}]=[\hat{a}, a(g)] \cup[b(g), \hat{b}]
$$

(ii) For $g<g_{0}$, we have $a(g)<b(g)$ and $(a(g), b(g))$ is a gap in the spectrum of the random operator $A_{g}$, located inside the gap $(a, b)$ of the unperturbed periodic operator $A_{0}$ Moreover, we have

$$
a \leqq a\left(1+g U_{+}\right)^{\frac{\iota}{l_{+}}} \leqq a(g) \leqq \frac{a}{1-g U_{+}}
$$

and

$$
b\left(1-g U_{+}\right) \leqq b(g) \leqq \frac{b}{\left(1+g U_{+}\right)^{\frac{l_{-}}{U_{+}}}} \leqq b .
$$

(iii) If $g_{0}<\frac{1}{U_{+}}$, we have $a(g)=b(g)$ for all $g \in\left[g_{0}, \frac{1}{U_{+}}\right)$, and the random operator $A_{g}$ has no gap inside the gap $(a, b)$ of the unperturbed periodic operator $A_{0}$, ie, $[\hat{a}, \hat{b}] \subset \Sigma_{g}$

Definition 4 (Exponential localization). We say that the random operator $A_{4}$ exhibits localization in an interval $I \subset \Sigma_{g}$, if $A_{g}$ has only pure point spectrum in $I$ with probability one We have exponential localization in $I$ if we have localization and, with probability one, all the eigenfunctions corresponding to eigenvalues in I have exponential decay 
Remark 5 The gradients of exponentially decaying eigenfunctions of $A_{g}$ always have exponentially decaying local $L^{2}$-norms (Corollary 40) Thus the corresponding energy densities (given by either (6) or (7)) also have exponentially decaying local $L^{2}$-norms, uniformly in the time $t$

Our main results show that random perturbations create exponentially localized eigenfunctions near the edges of the gap Our method requires low probability of extremal values for the random variables, the following two theorems achieve this in different ways The results are formulated for the left edge of the gap, with similar results holding at the right edge

Theorem 6 (Localization at the edge). Let the random operator $A_{g}$ defined by (18) satisfy Assumptions 1 and 2, with

$$
\mu\{(1-\gamma, 1]\} \leqq K \gamma^{\eta} \quad \text { for } 0 \leqq \gamma \leqq 1,
$$

where $K<\infty$ and $\eta>d$ For any $g<g_{0}$ there exists $\delta(g)>0$, depending only on the constants $d, g, q, \varrho_{0, \pm}, U_{ \pm}, r_{u}, a, b, K, \eta,\|\rho\|_{\infty}$, such that the random operator $A_{g}$ exhibits exponential localization in the interval $[a(g)-\delta(g), a(g)]$

Theorem 7 (Localization in a specified interval). Let the random operator $A_{g}$ defined by (18) satisfy Assumptions 1 and 2 For any $g<g_{0}$, given $a<a_{1}<a_{2}<a(g)$, with $a(g)-a_{1} \leqq b(g)-a(g)$, there exists $p_{1}>0$, depending only on the constants $d, g, q, \varrho_{0, \pm}, U_{ \pm}, r_{u}, a$, an upper bound on $\|\rho\|_{\infty}$ and on the given $a_{1}, a_{2}$, such that if

$$
\mu\left(\left(\frac{g_{1}}{g}, 1\right]\right)<p_{1}
$$

where $g_{1}$ is defined by $a\left(g_{1}\right)=a_{1}$, the random operator $A_{g}$ exhibits exponential localization in the interval $\left[a_{2}, a(g)\right]$

Theorems 6 and 7 can be extended to the situation when the gap is totally filled by the spectrum of the random operator, we then establish the existence of an interval (inside the original gap) where the random acoustic operator exhibits exponential localization. Notice that the extension of Theorem 7 tells us that we can arrange for localization in as much of the gap as we want.

Theorem 8 (Localization at the meeting of the edges). Let the random operator $A_{g}$ defined by (18) satisfy Assumptions 1 and 2, with

$$
\mu\{(1-\gamma, 1]\}, \mu\{[-1,-1+\gamma)\} \leqq K_{\gamma^{\prime}} \quad \text { for } 0 \leqq \gamma \leqq 1
$$

where $K<\infty$ and $\eta>d$ Suppose $g_{0}<\frac{1}{U_{+}}\left(e g\right.$, if $\left.\left(\frac{b}{a}\right)^{\frac{l}{2 U_{-}}}<2\right)$, so the random operator $A_{g}$ has no gap inside $(a, b)$ for $g \in\left[g_{0}, \frac{1}{U_{+}}\right)$Then there exist $0<\varepsilon<$ $\frac{1}{U_{+}}-g_{0}$ and $\delta>0$, depending only on the constants $d, q, \varrho_{0, \pm}, U_{ \pm}, r_{u}, a, b, K, \eta$, $\|\rho\|_{\infty}$, such that the random operator $A_{g}$ exhibits exponential localization in the interval $\left[a\left(g_{0}\right)-\delta, a\left(g_{0}\right)+\delta\right]$ for all $g_{0} \leqq g<g_{0}+\varepsilon$ 
Theorem 9 (Localization in a specified interval in the closed gap). Let the random operator $A_{g}$ defined by (18) satisfy Assumptions 1 and 2 Suppose $g_{0}<\frac{1}{U_{+}}$ (eg , if $\left(\frac{b}{a}\right)^{\frac{U_{+}}{2 L_{-}}}<2$ ), so the random operator $A_{g}$ has no gap inside $(a, b)$ for $g \in\left[g_{0}, \frac{1}{U_{+}}\right)$Let $a<a_{1}<a_{2}<a\left(g_{0}\right)=b\left(g_{0}\right)<b_{2}<b_{1}<b$ be given For any

$g \in\left[g_{0}, \frac{1}{U_{+}}\right)$there exist $p_{1}, p_{2}>0$, depending only on the constants $d, g, q, \varrho_{0, \pm}$, $U_{ \pm}, r_{u}, a, b$, an upper bound on $\|\rho\|_{\infty}$ and on the given $a_{1}, a_{2}, b_{1}, b_{2}$, such that if

$$
\mu\left(\left(\frac{g_{1}}{g}, 1\right]\right)<p_{1}, \quad \mu\left(\left[-1,-\frac{g_{2}}{g}\right)\right)<p_{2},
$$

where $g_{1}$ and $g_{2}$ are defined by $a\left(g_{1}\right)=a_{1}$ and $b\left(g_{2}\right)=b_{1}$ (notice $\left.0<g_{1}, g_{2}<g_{0} \leqq g\right)$, the random operator $A_{g}$ exhibits exponential localization in the interval $\left[a_{2}, b_{2}\right]$

Theorems 8 and 9 are proved exactly as Theorems 6 and 7, respectively, taking into account both edges of the gap

Remark 10 The methods of this paper also apply to random Schrödinger operators on $\mathbb{R}^{d}$ They give a new proof of localization at the bottom of the spectrum, as in [HM, CH, Kp] For random perturbations of a periodic Schrödinger operator with a gap in the spectrum, we obtain the analogues of Theorems 3,6, 7,8 and 9, extending our results on the lattice $[\mathrm{FK} 1]$ to the continuum

Remark 11 Theorems 6 and 8 should be true without the extra hypotheses (23) and (25) They are used in conjunction with a Combes-Thomas argument to obtain the starting hypothesis for the multiscale analysis, in the proof of localization One may expect estimates similar to Lifshitz tails (e g., [PF]) for the density of states inside the gap, which would replace (23) and (25) in the proofs. This is how the starting hypothesis is obtained for random Schrödinger operators at the bottom of the spectrum $[\mathrm{HM}]$

Combes and Hislop have announced an improved Combes-Thomas argument inside a gap, they obtain a decay rate proportional to the square root of the product of the distances to the edges of the gap. With this result we would only need $\eta>\frac{d}{2}$ in Theorem 6 , but we would still need to require $\eta>d$ in Theorem 8

Theorem 3 is proved in Sect 4, the proof requires periodic operators and periodic boundary conditions, studied in Sect. 3. A Combes-Thomas argument for acoustic operators is given in Sect 2 Theorems 6 and 7 are proved in Sect 6 by multiscale analyses The required Wegner-type estimate is in Sect 5 The starting hypotheses are proved first for finite volume acoustic operators with periodic boundary condition, using a Combes-Thomas argument for operators with periodic boundary condition (Sect. 3) and Theorem 3. Appendix A contains a result on measurability of random operators, from which follows the desired measurability for the acoustic operators we study We collect some results on elliptic operators in Appendix B.

We adopt the following definitions and notations.

- For $x=\left(x_{1}, ., x_{d}\right) \in \mathbb{R}^{d}$ we let $|x|_{p}=\left(x_{1}^{p}+\quad+x_{d}^{p}\right)^{1} p$ for $1 \leqq p<\infty$, and $|x|_{\infty}=\max _{1 \leqq j \leqq d}\left|x_{j}\right|$. We set $|x|=|x|_{2}$ and $\|x\|=|x|_{\infty}$

- $\Lambda_{L}(x)=\left\{y \in \mathbb{R}^{d},\|y-x\|<\frac{L}{2}\right\}$ is the (open) cube of side $L$ centered at $x \in \mathbb{R}^{d}, \bar{\Lambda}_{L}(x)$ is the closed cube, and $\breve{\Lambda}_{L}(x)=\left\{y \in \mathbb{R}^{d},-\frac{L}{2} \leqq y_{l}-x_{i}<\frac{L}{2}, i=\right.$ $1, \quad, d\}$ the half-open/half-closed cube 
- $\chi_{\Lambda}$ is the characteristic function of the set $\Lambda$, we write $\chi_{r . L}=\psi_{\Lambda_{l}(r)}$.

- A function $f$ on $\mathbb{R}^{d}$ is called $q$-periodic for some $q>0$ if $f(x+q i)=f(x)$ for all $x \in \mathbb{R}^{d}$ and $i \in \mathbb{Z}^{d}$ $\lambda \Omega$

- A domain $\Omega$ is an open connected subset of $\mathbb{R}^{d}$, its boundary is denoted by

- $L^{p}(\Omega)$ is the space of measurable complex-valued functions $u(x), x \in \Omega$ with the norm $\|u\|_{p}=\|u\|_{p, \Omega}=\left[\int_{\Omega}|u(x)|^{p} d x\right]^{1 / p}$ We will often use the space $L^{2}(\Omega)$ and in this case we will write $\|u\|_{\Omega}$ for $\|u\|_{2, \Omega}$ If $\Omega=\mathbb{R}^{d}$ we may omit it from the notation

- $C^{1}(\Omega)$ is the linear space of continuously differentiable functions on the domain $\Omega, C_{0}^{1}(\Omega)$ is the subspace of functions with compact support

- $W^{1, p}(\Omega)$ is the Sobolev space of complex-valued functions $u(x), x \in \Omega$ with the norm $\|u\|_{1, p, \Omega}=\left[\|u\|_{p, \Omega}^{p}+\|\mid \nabla u\|_{p, \Omega}^{p}\right]^{1 / p}$

- $\mathscr{B}(\mathscr{X}, \mathscr{Y})$ is the Banach space of bounded operators from the normed space $\mathscr{X}$ to the normed space $\mathscr{Y} ; \mathscr{B}(\mathscr{X})=\mathscr{B}(\mathscr{X}, \mathscr{X})$.

- The domain, spectrum and adjoint of a linear operator $A$ are denoted by $\mathscr{Z}(A)$, $\sigma(A)$ and $A^{*}$, respectively.

- The domain of a quadratic form $\mathscr{A}$ is denoted by $\mathscr{D}(\mathscr{A})$

- For a complex number $z$ its conjugate is denoted by $z^{*}$.

\section{A Combes-Thomas Argument}

Let the operator $A$ be given by (10) If $z \notin \sigma(A)$, we write $R(z)=(A-z)^{-1}$

Lemma 12. Let the operator $A$ be given by (10) with (11) Then for any $z \notin \sigma(A)$, $n \in \mathbb{N}$ and $\ell>0$ we have

$$
\left\|\varkappa_{x, r} R(z)^{n} \chi_{1, t}\right\| \leqq\left(\frac{9}{\eta}\right)^{n} e^{(\sqrt{d} t / 4)} e^{-m_{z}|r-\eta|} \quad \text { for all } x, y \in \mathbb{R}^{d},
$$

with

$$
m_{z}=\frac{\eta}{4\left[\varrho_{-}^{-1}+|z|+\eta\right]},
$$

where $\eta=\operatorname{dist}(z, \sigma(A))$

Proof We start by defining the operators formally given by

$$
A_{a}=e^{a \cdot x} A e^{-a \cdot x}, \quad a \in \mathbb{R}^{d}
$$

as the closed densely defined operators uniquely defined by the corresponding quadratic forms More precisely, for each $a \in \mathbb{R}^{d}$ we define quadratic forms on $C_{0}^{l}\left(\mathbb{R}^{d}\right)$ by

$$
\mathscr{A}_{a}[\psi]=\left\langle\nabla e^{a \cdot r} \psi, \frac{1}{\varrho(x)} \nabla e^{-a \cdot r} \psi\right\rangle=\left\langle(\nabla+a) \psi, \frac{1}{\varrho(x)}(\nabla-a) \psi\right\rangle
$$


and

$$
\begin{aligned}
\mathscr{Q}_{a}[\psi] & =\mathscr{A}_{a}[\psi]-\mathscr{A}[\psi] \\
& =\left\langle\psi, \frac{1}{\varrho(x)} a \cdot \nabla \psi\right\rangle-\left\langle a \cdot \nabla \psi, \frac{1}{\varrho(x)} \psi\right\rangle-|a|^{2}\left\langle\psi, \frac{1}{\varrho(x)} \psi\right\rangle .
\end{aligned}
$$

Notice that

$$
\left|\left\langle\psi, \frac{1}{\varrho(x)} a \cdot \nabla \psi\right\rangle\right| \leqq \frac{1}{2}\left\{\left\langle\psi, \frac{1}{\varrho(x)} \psi\right\rangle+\left\langle a \cdot \nabla \psi, \frac{1}{\varrho(x)} a \cdot \nabla \psi\right\rangle\right\}
$$

and

$$
\left\langle a \cdot \nabla \psi, \frac{1}{\varrho(x)} a \cdot \nabla \psi\right\rangle \leqq|a|^{2} \mathscr{A}[\psi] .
$$

Thus, if $|a| \leqq 1$ we have

$$
\left|\mathscr{Z}_{a}[\psi]\right| \leqq|a| \mathscr{A}[\psi]+|a|(1-|a|) \varrho_{-}^{-1}\|\psi\|^{2} \text { for all } \psi \in C_{0}^{1}\left(\mathbb{R}^{d}\right) .
$$

We now require $|a|<1$ and use [Ka; Theorem VI 3 9] to conclude that $\mathscr{A}_{a}$ is a closable sectorial form and define $A_{a}$ as the unique $m$-sectorial operator associated with it. If in addition $z \notin \sigma(A)$ and

$$
\Omega \equiv 2\left\|\left(|a|(1-|a|) \varrho_{-}^{-1}+|a| A\right) R(z)\right\|<1,
$$

we can conclude that $z \notin \sigma\left(A_{a}\right)$ and

$$
\left\|R(z)-R_{a}(z)\right\| \leqq \frac{4 \Omega}{(1-\Omega)^{2}}\|R(z)\|,
$$

where $R_{a}(z)=\left(A_{a}-z\right)^{-1}$

Since

$$
\begin{gathered}
\Omega=2 \|\left(\left(|a|(1-|a|) \varrho_{-}^{-1}+|a| z\right) R(z)+|a| \|\right. \\
\left.\leqq 2|a|\left((1-|a|) \varrho_{-}^{-1}+|z|\right) \eta^{-1}+1\right) \leqq 2|a|\left(\left(\varrho_{-}^{-1}+|z|\right) \eta^{-1}+1\right),
\end{gathered}
$$

it suffices to take

$$
|a|<\frac{\eta}{2\left(\varrho_{-}^{-1}+|z|+\eta\right)}
$$

to ensure $\Omega<1$ In fact, we get $\Omega \leqq \frac{1}{2}$ for $|a| \leqq m_{z}$ (given by (28)), so

$$
\left\|R_{a}(z)\right\| \leqq\left(1+\frac{4 \Omega}{(1-\Omega)^{2}}\right)\|R(z)\| \leqq \frac{9}{\eta}
$$

Now let $x_{0}, y_{0} \in \mathbb{R}^{d}, n \in \mathbb{N}, \ell>0$, and take $a=\frac{m_{z}}{\left|x_{0}-y_{0}\right|}\left(x_{0}-y_{0}\right)$ We have

$$
\begin{aligned}
\chi_{\gamma_{0}, t} R(z)^{n} \chi_{v_{0}, \ell} & =\chi_{x_{0}, t} e^{-a \cdot x} R_{a}(z)^{n} e^{a \cdot{ }^{\prime}} \chi_{v_{0}, t} \\
& =e^{-m_{z}\left|r_{0}-v_{0}\right|} \chi_{\gamma_{0}, t} e^{-a \cdot\left(x-x_{0}\right)} R_{a}(z)^{n} e^{a \cdot\left(x-\nu_{0}\right)} \chi_{v_{0}, t},
\end{aligned}
$$


so

$$
\left\|\chi_{\gamma_{0},} R(z)^{n} \chi_{\gamma_{0}, f}\right\| \leqq\left(\frac{9}{\eta}\right)^{n}\left\|\chi_{\gamma_{0}, f} e^{-a \cdot\left(x-\gamma_{0}\right)}\right\|_{\infty}\left\|\chi_{\gamma_{0},} e^{a \cdot\left(r-\gamma_{0}\right)}\right\|_{\infty} e^{-m_{z}\left|x_{0}-\gamma_{0}\right|}
$$

Since

$$
\left\|\chi_{r_{0}, t} e^{ \pm a \cdot\left(r-x_{0}\right)}\right\|_{\infty} \leqq e^{\frac{\sqrt{d} t}{2}|a|}=e^{\frac{\sqrt{d} t}{2} m_{z}},
$$

and clearly $m_{z} \leqq \frac{1}{4}$, the theorem is proved.

The next lemma gives an exponential estimate for the gradient of the resolvent

Lemma 13. Let the operator $A$ be given by (10) with (11), and let $z \notin \sigma(A)$ with $\eta, m_{z}$ as in Lemma 12 Then $\nabla R(z): L^{2}\left(\mathbb{R}^{d}\right) \rightarrow L^{2}\left(\mathbb{R}^{d}, \mathbb{C}^{d}\right)$ is a bounded operator with

$$
\|\nabla R(z)\| \leqq \Theta_{1}\left(\frac{(1+|z|)}{\eta}+1\right),
$$

where $\Theta_{1}=\Theta\left(d, \varrho_{ \pm}, 1\right)$ is given in (239) Furthermore, for each $\ell>0$ we have

$$
\left\|\chi_{x, t} \nabla R(z) \chi_{v, t}\right\| \leqq \Theta_{1}(1+|z|) \frac{9}{\eta} e^{(3 \sqrt{d} f ; 4)} e^{-m_{z}|r-v|}
$$

for all $x, y \in \mathbb{R}^{d}$ with $|x-y| \geqq 2 \ell$

Proof For any $\psi \in L^{2}\left(\mathbb{R}^{d}\right)$ we have $R(z) \psi \in W^{1,2}\left(\mathbb{R}^{d}\right)$, since $R(z) \psi \in \mathscr{L}(A)$ and we have (11). The bound (46) is thus an immediate consequence of Proposition 41 and of the evident identity $A R(z) \psi=(I+z R(z))$ The proof of (47) is based upon Lemma 12 and Proposition 39 Let $\ell>0, x, y \in \mathbb{R}^{d}$ with $|x-y| \geqq 2 \ell$. For any $\psi \in L^{2}\left(\mathbb{R}^{d}\right)$ we can use Proposition 39 to obtain

$$
\begin{aligned}
& \left\|\chi_{r, \ell} \nabla R(z) \chi_{r, \ell} \psi\right\| \leqq \Theta_{1}\left\{\left\|\chi_{x, 3 \ell} R(z) \chi_{v, \ell} \psi\right\|+\left\|\chi_{r, 3 \ell} A R(z) \chi_{v, \ell} \psi\right\|\right\} \\
& \leqq \Theta_{1}\left\{(1+|z|)\left\|\chi_{r, 3,} R(z) \chi_{\nu, \gamma} \psi\right\|+\left\|\chi_{x, 3 \ell} \chi_{y, \gamma} \psi\right\|\right\} \\
& =\Theta_{1}(1+|z|)\left\|\chi_{r, 3 \ell} R(z) \chi_{v, \ell} \psi\right\|
\end{aligned}
$$

Thus

$$
\begin{aligned}
\left\|\chi_{r, t} \nabla R(z) \chi_{1, t}\right\| & \leqq \Theta_{1}(1+|z|)\left\|\chi_{r, 3 \ell} R(z) \chi_{,, t}\right\| \leqq \Theta_{1}(1+|z|)\left\|\chi_{r, 3 \ell} R(z) \chi_{\gamma, 3 \ell}\right\| \\
& \leqq \Theta_{1}(1+|z|) \frac{9}{\eta} e^{(3 \sqrt{d} t / 4)} e^{-m_{z}|x-1|}
\end{aligned}
$$

where we used Lemma 12

\section{Periodic Operators and Periodic Boundary Condition}

As in the matrix case [FK1, FK2], the (non-random) spectrum of random acoustic operators can be represented as the union of the spectra of finite volume acoustic operators with periodic boundary condition 
In this section we study acoustic operators in periodic media. We say that the acoustic operator $A$ given by (10) with (11) is $q$-periodic for some $q>0$ if $Q(x)$ is a $q$-periodic function In this section $A$ will always denote such an operator

31 Periodic boundary condition We start by defining the restriction of such $A$ to a cube with periodic boundary condition. Given a cube $\Lambda=\Lambda_{\ell}(x)$, where $x \in \mathbb{R}^{d}$ and $\ell>0$; we will denote by $\AA$ the torus we obtain by identifying the edges of the closed cube $\bar{\Lambda}$ in the usual way. We introduce the usual distance in the torus

$$
\stackrel{i}{d}(x, y) \equiv \min _{m \in \ell \mathbb{Z}^{d}}|x-y+m| \leqq \frac{\sqrt{d} \ell}{2} \text { for all } x, y \in \bar{\Lambda}
$$

We will identify functions on $\AA$ with their $\ell$-periodic extensions to $\mathbb{R}^{d}$, for example, $C^{1}(\AA)$ will be identified with the space of continuously differentiable $\ell$-periodic functions on $\mathbb{R}^{d}$ We define $W^{1,2}(\AA)$ as the closure of $C^{1}(\AA)$ in $W^{1,2}(\Lambda)$

We will always take $\ell \in q \mathrm{~N}$ and define $\AA_{A}$, the restriction of $A$ to $\Lambda$ with periodic boundary condition, as the unique nonnegative self-adjoint operator on $L^{2}(\AA) \cong$ $L^{2}(\Lambda)$, defined by the nonnegative densely defined closed quadratic form

$$
\mathscr{\mathscr { L }}_{\Lambda}(\psi)=\left\langle\nabla \psi, \frac{1}{\varrho(x)} \nabla \psi\right\rangle \equiv \sum_{j=1}^{d}\left\langle\partial_{j} \psi, \frac{1}{\varrho(x)} \partial_{j} \psi\right\rangle, \quad \text { with } \psi \in W^{1,2}(\grave{\Lambda}),
$$

the inner product being in $L^{2}(\Lambda)$. It follows from (11) that $\AA_{\Lambda} \geqq-\frac{1}{Q_{+}} \dot{\Delta}_{\Lambda}$, where $\AA_{\Lambda}$ is the Laplacian with periodic boundary condition on $\Lambda$ Since $-\dot{\Delta}_{\Lambda}$ has compact resolvent, using the min-max principle (see [RS4, Theorem XIII.2]) we conclude that $\AA_{A}$ has compact resolvent.

The shift operators $U^{y}, y \in \mathbb{R}^{d}$ are defined by

$$
U^{v} \varphi(x)=\varphi(x-y), \quad \text { for all } x \in \mathbb{R}^{d}
$$

They are unitary operators in $L^{2}\left(\mathbb{R}^{d}\right)$, and if $m \in q \mathbb{Z}^{d}$ we have $U^{m} A U^{-m}=A$ (as unbounded operators), since $\varrho(x)$ is a $q$-periodic function Using the identification of functions on the torus with periodic functions, it is easy to see that for each $y \in \mathbb{R}^{d}$ we have that $U^{v}$ is a unitary operator from $L^{2}\left(\AA_{f}(x)\right)$ to $L^{2}\left(\AA_{f}(x+y)\right)$, for any $x \in \mathbb{R}^{d}$, and

$$
U^{v} \AA_{\Lambda,(x)} U^{-v}=\AA_{\Lambda,(x+v)}, \quad \text { so } \sigma\left(\AA_{\Lambda_{1}(r)}\right)=\sigma\left(\AA_{\Lambda_{\gamma}(x+v)}\right)
$$

32 Spectrum of periodic operators If $k, n \in \mathbb{N}$, we say that $k \preceq n$ if $n \in k \mathbb{N}$ and that $k \prec n$ if $k \preceq n$ and $k \neq n$.

Theorem 14. Suppose the operator A given by (10) with (11) is q-periodic Let $\left\{\ell_{n}, n=0,1,2, \quad\right\}$ be a sequence in $\mathbb{N}$ such that $\ell_{0}=q$ and $\ell_{n} \prec \ell_{n+1}$ for each $n=0,1,2$, . Then

$$
\sigma\left(\AA_{\Lambda_{\ell_{n}}(0)}\right) \subset \sigma\left(\AA_{\Lambda_{\ell_{n}+1}(0)}\right) \subset \sigma(A) \text { for all } n=0,1,2, .
$$


and

$$
\sigma(A)=\overline{\bigcup_{n \geqq 1} \sigma\left(\AA_{\Lambda_{\ell n}(0)}\right)}
$$

Notice that it follows from (56) that a gap in the spectrum of $A$ is inside a gap in the spectrum of $\AA_{1,(x)}$ for any $x \in \mathbb{R}^{d}$ and $\ell \in q \mathbb{N}$

A proof of (57) for periodic Schrödinger operators can be found in [Ea], based on Floquet theory This proof can be adapted to operators as in the theorem in the case of smooth coefficients For the nonsmooth coefficients we are interested in some aspects of the Floquet theory have to be revised We give an alternative proof which does not use Floquet theory.

To prove the theorem we will relate the resolvent $\stackrel{\circ}{R}_{A}(z)=\left(\stackrel{\circ}{A}_{A}-z I\right)^{-1}$, a bounded operator on $L^{2}(\Lambda)$, to the resolvent $R(z)=(A-z I)^{-1}$, a bounded operator on $L^{2}\left(\mathbb{R}^{d}\right)$ Let us fix a cube $\Lambda=\Lambda_{f}(x)$ for some $x \in \mathbb{R}^{d}$ and $\ell \in q \mathbb{N}$, the map

$$
\varphi \in L^{2}\left(\mathbb{R}^{d}\right) \mapsto \widetilde{\varphi}_{\Lambda}=\left\{\widetilde{\varphi}_{\Lambda, m}, m \in \ell \mathbb{Z}^{d}\right\} \in \ell^{2}\left(\ell \mathbb{Z}^{d}, L^{2}(\Lambda)\right),
$$

where

$$
\widetilde{\varphi}_{\Lambda, m}=\chi_{\Lambda}\left(U^{-m} \varphi\right)=U^{-m}\left(\chi_{1+m} \varphi\right) \text { for each } m \in \ell \mathbb{Z}^{d},
$$

establishes a unitary isomorphism between the Hilbert spaces $L^{2}\left(\mathbb{R}^{d}\right)$ and $\ell^{2}\left(\ell \mathbb{Z}^{d}, L^{2}(\Lambda)\right.$ ) Under this unitary map, bounded operators $T$ on $L^{2}\left(\mathbb{R}^{d}\right)$ have the following matrix representation on $\ell^{2}\left(\ell \mathbb{Z}^{d}, L^{2}(\Lambda)\right)$

$$
\widetilde{T}_{\Lambda}=\left\{\widetilde{T}_{\Lambda, m, n}=\chi_{\Lambda} U^{-m} T U^{n} \chi_{\lambda,}, m, n \in \ell \mathbb{Z}^{d}\right\},
$$

where each $\widetilde{T}_{1, m, n}$ is a bounded operator on $L^{2}(\Lambda)$ If $R$ is a bounded $\ell$-periodic operator, i e, $U^{-m} R U^{m}=T$ for all $m \in \ell \mathbb{Z}^{d}$, we have

$$
\widetilde{R}_{1, m, n}=\widehat{R}_{\Lambda, n-m} \equiv \chi_{\Lambda} R U^{n-m} \chi_{\Lambda}
$$

so $\widetilde{R}_{1}$ is a Toeplitz matrix with operator valued entries $\widehat{R}_{\Lambda, n-m}$ Notice that $R$ is uniquely determined by

$$
\widehat{R}_{A}=\left\{\widehat{R}_{A . m}, m \in \ell \mathbb{Z}^{d}\right\}
$$

We now introduce the Banach spaces

$$
\begin{aligned}
\mathscr{L}_{A}= & \left\{\varphi \in L^{2}\left(\mathbb{R}^{d}\right), \widetilde{\varphi}_{\Lambda} \in \ell^{1}\left(\ell \mathbb{Z}^{d}, L^{2}(\Lambda)\right)\right\}, \\
& \text { with }\|\varphi\|_{\mathscr{L}_{1}}=\left\|\widetilde{\varphi}_{1}\right\|_{/ 1 /\left(/ \mathbb{Z}^{d}, L^{2}(\Lambda)\right)},
\end{aligned}
$$

and

$$
\begin{aligned}
\mathscr{H}_{1}= & \left\{\varphi \in W^{1,2}\left(\mathbb{R}^{d}\right), \varphi,|\nabla \varphi| \in \mathscr{L}_{1}\right\}, \\
& \text { with }\|\varphi\|_{\mathscr{H}_{1}}^{2}=\|\varphi\|_{\mathscr{L}_{1}}^{2}+\|\mid \nabla \varphi\|_{\mathscr{L}_{1}}^{2},
\end{aligned}
$$


and the normed space

$$
\begin{aligned}
\mathscr{B}_{\Lambda}= & \left\{R \in \mathscr{B}\left(L^{2}\left(\mathbb{R}^{d}\right)\right) \ell \text {-periodic, } \widehat{R}_{\Lambda} \in \ell^{1}\left(\ell \mathbb{Z}^{d}, \mathscr{B}\left(L^{2}(\Lambda)\right)\right)\right\}, \\
& \text { with }\|R\|_{\mathscr{B}_{1}}=\left\|\widehat{R}_{\Lambda}\right\|_{\ell^{1}\left(\ell \mathbb{Z}^{d}, \mathscr{B}\left(L^{2}(\Lambda)\right)\right)}
\end{aligned}
$$

We define the maps

$$
\varphi \in \mathscr{L}_{\Lambda} \mapsto P_{\Lambda} \varphi=\sum_{m \in / \mathbb{Z}^{d}} \widetilde{\varphi}_{\Lambda . m}=\chi_{\Lambda} \sum_{m \in / \mathbb{Z}^{d}} U^{-m} \varphi \in L^{2}(\Lambda)
$$

and

$$
R \in \mathscr{B}_{\Lambda} \mapsto \mathscr{P}_{\Lambda}(R)=\sum_{m \in \ell \mathbb{Z}^{d}} \widehat{R}_{\Lambda, m}={\chi_{\Lambda}} R \sum_{m \in \ell \mathbb{Z}^{d}} U^{m} \chi_{\Lambda} \in \mathscr{B}\left(L^{2}(\Lambda)\right)
$$

Lemma 15. The maps $P_{A} \cdot \mathscr{L}_{\Lambda} \rightarrow L^{2}(\Lambda)$ and $\mathscr{P}_{\Lambda} . \mathscr{B}_{\Lambda} \rightarrow \mathscr{B}\left(L^{2}(\Lambda)\right)$ are linear contractions, with $P_{A}$ being onto Moreover

(i)

$$
\mathscr{B}_{A} \subset \mathscr{B}\left(\mathscr{L}_{A}\right) \text { with }\|R\|_{\mathscr{B}\left(\mathscr{L}_{1}\right)} \leqq\|R\|_{\mathscr{M}_{1}} \text { for all } R \in \mathscr{B}_{1} \text {, }
$$

and

$$
P_{\Lambda} R \varphi=\mathscr{P}_{\Lambda}(R) P_{\Lambda} \varphi \quad \text { for all } R \in \mathscr{B}_{\Lambda}, \varphi \in \mathscr{L}_{\Lambda}
$$

(ii)

$$
R S \in \mathscr{B}_{\Lambda} \quad \text { and } \mathscr{P}_{\Lambda}(R S)=\mathscr{P}_{\Lambda}(R) \mathscr{P}_{\Lambda}(S) \text { for all } R, S \in \mathscr{B}_{\Lambda}
$$

(iii)

$$
P_{1}\left(\mathscr{H}_{\Lambda}\right)=W^{1,2}(\stackrel{\circ}{)}
$$

and

$$
\nabla P_{\Lambda} \varphi=P_{1} \nabla \varphi \quad \text { for all } \varphi \in \mathscr{H}_{\Lambda}
$$

Proof We will prove (iii), the other statements in the lemma being straightforward We start by showing that

$$
P_{\Lambda}\left(C_{0}^{1}\left(\mathbb{R}^{d}\right)\right)=C^{1}(\stackrel{\curlywedge}{)}
$$

and

$$
\nabla P_{\Lambda} \varphi=P_{\Lambda} \nabla \varphi \quad \text { for all } \varphi \in C_{0}^{1}\left(\mathbb{R}^{d}\right)
$$

Let $\varphi \in C_{0}^{l}\left(\mathbb{R}^{d}\right)$, since it has compact support we have that $\sum_{m \in / \mathbb{Z}^{d}} U^{-m} \varphi$ is an $\ell$-periodic function in $C^{1}\left(\mathbb{R}^{d}\right)$ (the sum is locally finite), so $P_{\Lambda} \varphi \in C^{1}(\AA)$ and (74) holds by the definition of $P_{\Lambda}$. To see that we have equality in (73), it now suffices 
to notice that

$$
C_{\iota}^{\jmath}\left(\stackrel{\circ}{\Lambda} \equiv\left\{\varphi \in C^{\mathrm{l}}(\stackrel{\circ}{\Lambda}), \sup \{\dot{d}(x, y), x, y \in \operatorname{supp} \varphi\} \leqq \frac{\ell}{2}\right\} \subset P_{\Lambda}\left(C_{0}^{1}\left(\mathbb{R}^{d}\right)\right),\right.
$$

and any $\varphi \in C^{1}(\stackrel{i}{)}$ can be written as a finite linear combination of functions in $C_{c}^{1}(\stackrel{\circ}{\Lambda})$

Since $P_{A} \quad \mathscr{L}_{A} \rightarrow L^{2}(\Lambda)$ is a contraction, it follows from (73) and (74) that $P_{A}$ is also a contraction from $C_{0}^{1}\left(\mathbb{R}^{d}\right)$, equipped with the norm of $\mathscr{H}_{\Lambda}$, to $C^{1}(\dot{\Lambda})$, with the norm of $W^{1,2}(\AA)$ As $C_{0}^{1}\left(\mathbb{R}^{d}\right)$ and $C^{1}(\AA)$ are dense in $\mathscr{H}_{i}$ and $W^{1,2}(\AA)$, respectively, we can conclude that $P_{A}$ is a contraction from $\mathscr{H}_{A}$ to $W^{1,2}(\AA)$, with dense range because of (73), and (72) holds To show the equality in (71), define $W_{c}^{1,2}(\AA)$ as the

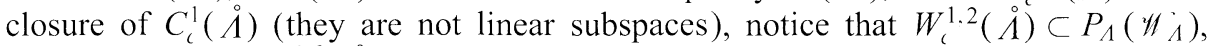
and that any $\varphi \in W^{1,2}(\AA)$ can be written as a finite linear combination of functions in $W_{i}^{1,2}(\stackrel{i}{)}$

For each $N \in \mathbb{N}$ we define the operator

$$
W_{N}=\sum_{m \in / \mathbb{Z}^{d},|m| \leqq N} U^{-m}
$$

Lemma 16. (i) $W_{N} \in \mathscr{B}\left(\mathscr{L}_{\Lambda}\right)$ for each $N \in \mathbb{N}$ and for all $\psi, \varphi \in \mathscr{L}_{\Lambda}$ we have

$$
\lim _{N \rightarrow \infty}\left\langle\psi, W_{N} \varphi\right\rangle_{L^{2}\left(\mathbb{R}^{d}\right)}=\left\langle P_{\Lambda} \psi, P_{\Lambda} \varphi\right\rangle_{L^{2}(\Lambda)}
$$

(ii) $W_{N} \in \mathscr{B}\left(\mathscr{H}_{\Lambda}\right)$ for each $N \in \mathbb{N}$ and for all $\psi, \varphi \in \mathscr{H}_{\Lambda}$ we have

$$
\lim _{N \rightarrow \infty} \mathscr{A}\left(\psi, W_{N} \varphi\right)=\mathscr{A}_{\Lambda}\left(P_{\Lambda} \psi, P_{\Lambda} \varphi\right)
$$

Proof (i) and the fact that $W_{N} \in \mathscr{B}\left(\mathscr{W}_{A}\right)$ follow easily from the definitions To prove (78), let $\psi, \varphi \in \mathscr{H}_{1}$, we have

$$
\mathscr{A}\left(\psi, W_{N} \varphi\right)=\left\langle\nabla \psi, \frac{1}{\varrho(x)} \nabla W_{N} \varphi\right\rangle_{L^{2}\left(\mathbb{R}^{d}\right)}=\left\langle\nabla \psi, W_{N} \frac{1}{\varrho(x)} \nabla \varphi\right\rangle_{L^{2}\left(\mathbb{R}^{d}\right)},
$$

where we used the $\ell$-periodicity of $\varrho(x)$ Since $\nabla \psi, \frac{1}{\varrho(r)} \nabla \varphi \in \mathscr{L}_{1}$, we can use first (77) and then Lemma 15(iii) to get

$$
\begin{aligned}
\lim _{N \rightarrow \infty} & \mathscr{A}\left(\psi, W_{N} \varphi\right)=\left\langle P_{\Lambda} \nabla \psi, P_{\Lambda} \frac{1}{\varrho(x)} \nabla \varphi\right\rangle_{L^{2}(\Lambda)} \\
=\left\langle\nabla P_{\Lambda} \psi, \frac{1}{\varrho(x)} \nabla P_{\Lambda} \varphi\right\rangle_{L^{2}(\Lambda)} & =\mathscr{A}_{\Lambda}\left(P_{\Lambda} \psi, P_{\Lambda} \varphi\right)
\end{aligned}
$$


Lemma 17. Suppose $z \notin \sigma(A)$, then

(i) $R(z) \in \mathscr{B}_{A} \cap \mathscr{B}\left(\mathscr{L}_{\Lambda}, \mathscr{H}_{A}\right)$

(ii) $z \notin \sigma\left(\AA_{A}\right)$ and

$$
\stackrel{\circ}{R}_{\Lambda}(z)=\mathscr{P}_{\Lambda}(R(z))
$$

Proof Let us fix $z \notin \sigma(A), R(z)$ is then a bounded $\ell$-periodic operator since $A$ is $q$-periodic and $\ell \in q \mathbb{N}$ Using Lemmas 12 and 13 we get $R(z) \in \mathscr{B}_{A}$ and $R(z) \in \mathscr{B}\left(\mathscr{L}_{\Lambda}, \mathscr{H}_{\Lambda}\right)$

Now let $\varphi \in \mathscr{L}_{\Lambda}, \psi \in \mathscr{H}_{\Lambda}$, then $R(z) \varphi \in \mathscr{H}_{\Lambda}$ and, by Lemma $15, P_{\Lambda} \psi, P_{\Lambda} R(z) \varphi$ $=\mathscr{P}_{\Lambda}(R(z)) P_{\Lambda} \varphi \in W^{1.2}(\AA)$ Thus we can use Lemma 16 and the identity $A R(z)=$ $z R(z)+I$ to obtain

$$
\begin{aligned}
& \mathscr{\mathscr { A }}_{\Lambda}\left(P_{\Lambda} \psi, \mathscr{P}_{\Lambda}(R(z)) P_{\Lambda} \varphi\right)=\mathscr{\mathscr { A }}_{\Lambda}\left(P_{\Lambda} \psi, P_{\Lambda} R(z) \varphi\right)=\lim _{N \rightarrow \infty} \mathscr{A}\left(\psi, W_{N} R(z) \varphi\right) \\
& =\lim _{N \rightarrow \infty} \mathscr{A}\left(\psi, R(z) W_{N} \varphi\right)=\left\langle\psi,(z R(z)+I) W_{N} \varphi\right\rangle_{L^{2}\left(\mathbb{R}^{d}\right)} \\
& =\left\langle\psi, W_{N}(z R(z)+I) \varphi\right\rangle_{L^{2}\left(\mathbb{R}^{d}\right)}=\left\langle P_{\Lambda} \psi, P_{\Lambda}(z R(z)+I) \varphi\right\rangle_{L^{2}(\Lambda)} \\
& =\left\langle P_{\Lambda} \psi,\left(z \mathscr{P}_{\Lambda}(R(z))+I\right) P_{\Lambda} \varphi\right\rangle_{L^{2}(\Lambda)}
\end{aligned}
$$

Recalling that $P_{\Lambda}\left(\mathscr{L}_{\Lambda}\right)=L^{2}(\Lambda)$ and $P_{\Lambda}\left(\mathscr{H}_{\Lambda}\right)=W^{1,2}(\stackrel{\AA}{)}$ (see Lemma 15), we can rewrite $(82)$ as

$$
\begin{aligned}
\mathscr{L}_{\Lambda}\left(\psi, \mathscr{P}_{\Lambda}(R(z)) \varphi\right)= & \left\langle\psi,\left(z \mathscr{P}_{\Lambda}(R(z))+I\right) \varphi\right\rangle_{L^{2}(\Lambda)} \\
& \text { for all } \varphi \in L^{2}(\Lambda), \psi \in W^{1,2}(\stackrel{\AA}{)})
\end{aligned}
$$

We can now conclude that $\mathscr{P}_{\Lambda}(R(z)) \varphi \in \mathscr{Z}\left(\dot{A}_{\Lambda}\right)$ for all $\varphi \in L^{2}(\Lambda)$, and

$$
\left(\AA_{\Lambda}-z I\right) \mathscr{P}_{\Lambda}(R(z)) \varphi=\varphi \text { for all } \varphi \in L^{2}(\Lambda) \text {. }
$$

If $z \notin \sigma\left(\AA_{A}\right)$ we can immediately conclude that $\stackrel{\circ}{R}_{A}(z)=\mathscr{P}_{\Lambda}(R(z))$. Thus if $\operatorname{Im} z \neq 0$, we are done. If $z \notin \sigma(A)$ and $z$ is real, notice that it follows from (77), (69) and the $\ell$ - periodicity of $R(z)$ that $\mathscr{P}_{\Lambda}(R(z))$ is a bounded self-adjoint operator Since $\AA_{1}$ is self-adjoint, it follows from (84) by taking adjoints that we also have

$$
\mathscr{P}_{\Lambda}(R(z))\left(\AA_{\Lambda}-z I\right) \varphi=\varphi \text { for all } \varphi \in \mathscr{L}\left(\AA_{\Lambda}\right) \text {, }
$$

hence $z \notin \sigma\left(\AA_{\Lambda}\right)$ and $\stackrel{\circ}{R}_{\Lambda}(z)=\mathscr{P}_{\Lambda}(R(z))$

Proof of Theorem 14 We already proved that $\sigma\left(\AA_{\Lambda_{f}(r)}\right) \subset \sigma(A)$ in Lemma 17, for all $x \in \mathbb{R}^{d}$ and $\ell \succ q$ To complete the proof of (56), it suffices to notice that if $q \prec \ell \prec L$, the theory developed in this section, up to and including Lemma 17 , can be applied with $\Lambda=\Lambda_{\ell}(0)$ and with the torus $\AA_{L}(0)$ substituted for $\mathbb{R}^{d}$, with the obvious modifications

Thus it only remains to show that

$$
\sigma(A) \subset \Sigma \equiv \overline{\bigcup_{n \geqq 1} \sigma\left(\AA_{\Lambda_{\prime \prime}(0)}\right)}
$$


We only have to worry about real $E$ So let $E$ be real, with $E \notin \Sigma$, and let $\delta \equiv d(E, \Sigma)>0 \quad$ We have

$$
\left\|{\stackrel{\circ}{\Lambda_{\Lambda_{\ell}(0)}}}_{(E+i \eta)}\right\| \leqq \frac{1}{\delta} \quad \text { for all } n=1,2, \quad \text {. and } \eta \in \mathbb{R}
$$

Now let $\varphi$ be an arbitrary function in $L^{2}\left(\mathbb{R}^{d}\right)$ with compact support It follows that for all sufficiently large $n$ we have $\varkappa_{\Lambda_{/ n}(0)} \varphi=\varphi$, so $\widetilde{\varphi}_{\Lambda_{/ n}(0), m}=0$ for all $m \in \ell \mathbb{Z}^{d}, m \neq 0$ and $\widetilde{\varphi}_{\Lambda_{\ell_{n}}(0) .0}=\chi_{\Lambda_{\Lambda_{n}(0)}} \varphi=\varphi$ Thus it follows from (81) that

$$
\stackrel{\circ}{R}_{\Lambda_{\ell n}(0)}(z) \varphi=\chi_{\Lambda_{/ n}(0)} R(z) \varphi+\chi_{\Lambda_{\lambda_{n}}(0)} R(z) \sum_{m \in \ell_{n} \mathbb{Z}^{d}, m \neq 0} U^{m} \chi_{\Lambda_{\lambda_{n}}(0)} \varphi,
$$

for all $z=E+i \eta, \eta \neq 0$ and sufficiently large $n$ Using Lemma 12 we can verify that

$$
\lim _{n \rightarrow \infty}\left\|\chi_{\Lambda_{/ n}(0)} R(z) \sum_{m \in t_{n} \mathbb{Z}^{d}: m \neq 0} U^{m} \chi_{\Lambda_{/ n}(0)}\right\|=0
$$

so it follows from $(87)$ and $(88)$ that

$$
\|R(z) \varphi\|=\lim _{n \rightarrow \infty}\left\|\chi_{\Lambda_{\iota_{n}}(0)} R(z) \varphi\right\| \leqq \frac{1}{\delta}\|\varphi\| \text { for all } z=E+i \eta, \eta \neq 0
$$

Since functions with compact support are dense in $L^{2}\left(\mathbb{R}^{d}\right)$, we conclude that

$$
\|R(z)\| \leqq \frac{1}{\delta} \quad \text { for all } z=E+i \eta, \eta \neq 0
$$

Since $A$ is self-adjoint, we have $E \notin \sigma(A)$

\section{A Combes-Thomas argument for the torus}

Lemma 18. Let the operator A given by (10) with (11) be q-periodic, and let $\Lambda=\Lambda,\left(x_{0}\right)$ for some $x_{0} \in \mathbb{R}^{d}$ and $\ell \in q \mathbb{N}, \ell>2 r+8$, where $r>0$ Then for any $z \notin \sigma\left(\dot{A}_{\Lambda}\right)$ and $n \in \mathbb{N}$ we have

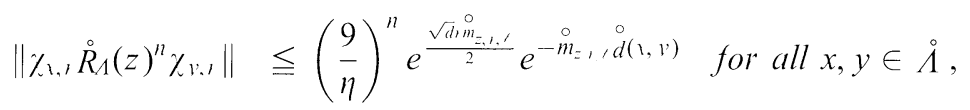

with

$$
\stackrel{\circ}{m}_{z, l, l}=\frac{\eta}{4\left(\frac{2 \sqrt{d}}{1-\frac{2 l+8}{l}}+1\right)\left[\varrho_{-}^{-1}+|z|+\eta\right]},
$$

where $\eta=\operatorname{dist}\left(z, \sigma\left(\AA_{A}\right)\right)$

Proof Let us fix $x_{1}, y_{1} \in \bar{\Lambda}$, changing the representation of the torus $\AA$ by a shift (see (55) and the discussion preceding it), we can assume $x_{0}=\frac{1}{2}\left(x_{1}+y_{1}\right)$ and $x_{1}, y_{1} \in \overline{\Lambda_{/ 12}\left(x_{0}\right)}$ In particular, $\dot{d}\left(x_{1}, y_{1}\right)=\left|x_{1}-y_{1}\right|$ Let $\ell>2 r+8$, we pick a real valued function $\xi \in C_{0}^{1}(\mathbb{R})$ with $0 \leqq \zeta(t) \leqq 1$ for all $t \in \mathbb{R}$, such that $\xi(t)=1$ for $|t| \leqq \frac{1}{4}+\frac{1}{2}, \xi(t)=0$ for $|t| \geqq \frac{t}{2}-1$, and $\left|\xi^{\prime}(t)\right| \leqq\left(\frac{t}{4}-\frac{1}{2}-2\right)^{-1}$ for all $t \in \mathbb{R}$ We set $\Xi(x)=\prod_{l=1}^{d} \xi\left(x_{l}\right)$ for $x \in \mathbb{R}^{d}$. Notice $\operatorname{supp} \Xi\left(x-x_{0}\right) \subset \Lambda$ 
We now proceed as in the proof of Lemma 12 with $\Lambda$ substituted for $\mathbb{R}^{d}$ and definition (29) replaced by

$$
\left(\AA_{\Lambda}\right)_{a}=e^{\Xi\left(x-r_{0}\right) a \cdot\left(r-r_{0}\right)} \AA_{\Lambda} e^{-\Xi\left(r-r_{0}\right) a \cdot\left(r-r_{0}\right)}, \quad a \in \mathbb{R}^{d}
$$

Notice that

$$
\begin{aligned}
\left|\nabla\left[\Xi\left(x-x_{0}\right) a \cdot\left(x-x_{0}\right)\right]\right| & \leqq\left(\frac{\left(\frac{t}{2}-1\right) \sqrt{d}}{\frac{t}{4}-\frac{t}{2}-2}+1\right)|a| \\
& \leqq\left(\frac{2 \sqrt{d}}{1-\frac{2 \imath+8}{t}}+1\right)|a| \text { for all } x \in \Lambda
\end{aligned}
$$

We can thus repeat the proof of Lemma 12, except that we must now require that $\left(\frac{2 \sqrt{d}}{1-\frac{21+8}{t}}+1\right)|a|<1$, and we must substitute $\left(\frac{2 \sqrt{d}}{1-\frac{21+8}{t}}+1\right)|a|$ for $|a|$ in (36) and in the rest of the proof Thus if $\left(\frac{2 \sqrt{d}}{1-\frac{2 r+8}{d}}+1\right)|a| \leqq m_{z}$, i e, $|a| \leqq m_{z, t, t}$, we have the equivalent of (41) We thus choose

$$
a=\frac{\stackrel{\circ}{m_{z, t,}}}{\left|x_{1}-y_{1}\right|}\left(x_{1}-y_{1}\right),
$$

and complete the proof of as before (with $x_{1}, y_{1}$ substituted for $x, y$ in (27)), as $\chi_{x_{1}} e^{-\Xi\left(r-r_{0}\right) a \cdot\left(x-x_{0}\right)}=\chi_{x_{1}} e^{-a \cdot\left(r-r_{0}\right)}$ and $\chi_{v_{1}} e^{\Xi\left(x-r_{0}\right) a \cdot\left(x-\gamma_{0}\right)}=\chi_{v_{1}} e^{a \cdot\left(r-x_{0}\right)}$

\section{Location of the Spectrum of Random Operators}

In this section we prove Theorem 3.

41 Approximation by periodic operators Let us fix a disorder parameter $g$ In order to investigate the samples of the random quantity $\varrho_{g, \omega}(x)$ we set

$$
\begin{gathered}
\mathscr{T}_{g}=\left\{\tau \quad \tau=\left\{\tau_{l}, i \in \mathbb{Z}^{d}\right\},-g \leqq \tau_{i} \leqq g\right\}, \\
\mathscr{T}_{g}^{(n)}=\left\{\tau \in \mathscr{T} \cdot \tau_{l+n l}=\tau_{i} \text { for all } i, j \in \mathbb{Z}^{d}\right\}, \quad n \in \mathbb{N},
\end{gathered}
$$

and

$$
\mathscr{T}_{g}^{(\infty)}=\bigcup_{n \succeq q} \mathscr{T}_{y}^{(n)}
$$

For $\tau \in \mathscr{T}_{y}$ we let

$$
\varrho_{\tau}(x)=\varrho_{0}(x)\left[1+\sum_{l \in \mathbb{Z}^{d}} \tau_{l} u(x-i)\right]
$$

and

$$
A(\tau)=A\left(\varrho_{\tau}\right)
$$

In addition, we set

$$
\mathscr{E}_{g}^{(n)}=\left\{\varrho_{\tau} \cdot \tau \in \mathscr{T}_{g}^{(n)}\right\} \quad \text { and } \quad \mathscr{E}_{G}^{(\infty)}=\bigcup_{n \succeq q} \mathscr{E}_{\mathscr{G}}^{(n)}
$$


To approximate acoustic operators by periodic operators, given $\tau \in \tilde{T}_{g}, n \in \mathbb{N}$ and $x \in \mathbb{R}^{d}$, we specify $\tau_{1_{n}(\imath)} \in \mathscr{T}_{y}^{(n)}$ by requiring $\left(\tau_{\Lambda_{n}(x)}\right)_{l}=\tau_{l}$ for all $i \in \check{\Lambda}_{n}(x) \cap \mathbb{Z}^{d}$, and define

$$
A_{1_{1}(x)}(\tau)=A\left(\tau_{1_{n}(1)}\right)
$$

The following lemma shows that the (nonrandom) spectrum of the random acoustic operator $A_{y}$ is determined by the spectra of the periodic acoustic operators $A(\tau)$, $\tau \in \mathscr{T}_{\mathscr{G}}^{(\infty)}$ The analogous result for random Schrödinger operators was proven by Kirsch and Martinelli [KM2, Theorem 4]

Lemma 19. Let the random operator $A_{y}$ defined by (18) satisfy Assumption 1, and let

$$
\Sigma_{g}=\overline{\bigcup_{\tau \in \pi_{g}^{(x)}} \sigma(A(\tau))}
$$

Then $\sigma\left(A_{q}\right)=\Sigma_{q}$ with probability one

Proof We start by showing that

$$
\sigma(A(\tau)) \subset \Sigma_{y} \text { for all } \tau \in \mathscr{T}_{y}
$$

Let $\Lambda_{n}=\Lambda_{\prime \prime}(0)$, where $\left\{\ell_{n}, n=0,1,2, \quad\right\}$ is a sequence in $\mathbb{N}$ such that $\ell_{0}=q$ and $\ell_{n} \prec \ell_{n+1}$ for each $n=0,1,2, \quad$ Let us pick $\tau \in \mathscr{T}_{y}$, and consider the associated sequence of operators $A_{n}(\tau)=A_{A_{n}}(\tau)$ We define bounded nonnegative measurable functions

$$
0_{\tau, n}^{ \pm}(x)=\max \left\{ \pm\left(\frac{1}{\varrho_{\tau_{1_{n}}}(x)}-\frac{1}{\varrho_{\tau}(x)}\right), 0\right\}
$$

and corresponding self-adjoint operators

$$
\Theta_{n}^{ \pm}(\tau)=-\nabla \cdot 0_{\tau, n}^{ \pm}(x) \nabla
$$

defined by the corresponding quadratic forms as in (12) It is not hard to see that

$$
0 \leqq \Theta_{n}^{ \pm}(\tau) \leqq \alpha^{ \pm} A(\tau)
$$

with

$$
0 \leqq \alpha^{-}=1-\frac{\varrho_{y,-}}{\varrho_{g .+}}<1, \quad 0 \leqq \alpha^{+}=\frac{\varrho_{y \cdot+}}{\varrho_{g \cdot-}}-1<\infty
$$

and

$$
A(\tau)-\Theta_{n}^{-}(\tau) \leqq A_{n}(\tau) \leqq A(\tau)+\Theta_{n}^{+}(\tau),
$$

as quadratic forms $\mathscr{Z}=C_{0}^{1}\left(\mathbf{R}^{d}\right)$ is a core for $\sqrt{A(\tau)}$, and for any $\psi \in \mathscr{Z}$ we have $\left\langle\psi, \Theta_{n}^{ \pm}(\tau) \psi\right\rangle=0$ for sufficiently large $n$, since $\left(\varrho_{\tau_{\mu_{1 n}}}(x)-\varrho_{\tau}(x)\right) \%_{0,\left(\ell_{n}-4 l_{u^{\prime \prime}}\right)}(x)=0$ The last observation and (108)-(110) allow us to apply Lemma 45 and conclude that (105) is true

To prove the opposite inclusion to (105), with probability one, we introduce the countable set

$$
\mathscr{T}_{g}^{(\infty)}(\mathbb{Q})=\mathscr{T}_{g}^{(\infty)} \cap \mathbb{Q}^{\mathbb{Z}^{d}}
$$

Approximating any $\tau \in \mathscr{T}_{g}^{(\infty)}$ uniformly by a sequence $\tau_{n} \in \mathscr{T}_{g}^{(\infty)}(\mathbb{Q})$, and using Lemma 45 together with appropriate inequalities analogous to (108)-(110), one can 
show that

$$
\sigma(A(\tau)) \subset \overline{\bigcup_{\tau^{\prime} \in \pi_{\| 1}^{\prime(\infty)}(\mathbb{Q})} \sigma\left(A\left(\tau^{\prime}\right)\right)}
$$

which readily implies that

$$
\Sigma_{y}=\overline{\bigcup_{\tau \in \pi_{l}^{(x)}(\mathbb{Q})} \sigma(A(\tau))}
$$

Thus, to finish the proof of Lemma (19) it suffices to show that, with probability one, we have

$$
\sigma(A(\tau)) \subset \sigma\left(A_{g}\right) \text { for any } \tau \in \mathscr{T}_{g}^{(\infty)}(\mathbb{Q})
$$

Notice that $A_{g}=A_{(y \cdot())}=A(g(1))$, with $g(1)=\left\{g()_{i}, i \in \mathbb{Z}^{d}\right\} \in \mathscr{T}_{y}$, since $(1) \in \Omega \equiv$ $[-1,1]^{\mathbb{Z}^{d}}$ Let $\left\{t_{n}, n=0,1,2, \quad\right\}$ be a sequence in $\mathbb{N}$ such that $\ell_{0}=q$ and $l_{n} \prec l_{n+1}$ for each $n=0,1,2, \quad$ For each $n$ and $\tau \in \mathscr{T}_{g}^{\left(q^{\prime}\right)}(\mathbb{Q})$ for some $q^{\prime} \succeq q$, we consider the event

$$
\begin{aligned}
\Omega_{\tau . n}= & \left\{(1) \in \Omega, \max _{\left.i \in \mathcal{U}_{, n}\left(m_{c, ?}\right) \cap\right) \cap \mathbb{Z}^{d}}\left|g()_{i}-\tau_{l}\right| \leqq\left(\ell_{n}+1\right)^{-(d+1)}\right. \\
& \text { for some } \left.m_{(j, \tau, n} \in q^{\prime} \mathbb{Z}^{d}\right\},
\end{aligned}
$$

notice $\mathbb{P}\left(\Omega_{\tau, n}\right)=1$ We now take the countable intersection

$$
\widehat{\Omega}=\bigcap_{n=0}^{\infty} \bigcap_{q^{\prime} \succeq q} \bigcap_{\tau \in \pi^{\prime\left(y^{\prime}\right.}(\mathbb{Q})} \Omega_{\tau n},
$$

so we have $\mathbb{P}(\widehat{\Omega})=1$ We will show that (114) is true for any $(\omega) \in \widehat{\Omega}$

So let $(1) \in \widehat{\Omega}, \quad \tau \in \mathscr{T}_{g}^{\left(q^{\prime}\right)}(\mathbb{Q})$ for some $q^{\prime} \succeq q, n \in \mathbb{N}$, and let $m_{(1, \tau, n}$ be as in (115) We set $(1)^{(n)}=\left\{(1)_{l}^{(n)}=\left(0_{l-m_{l, \tau}, n}, i \in \mathbb{Z}^{d}\right\}\right.$, and notice that $\sigma\left(A_{\left(g_{1}()^{(n)}\right)}\right)=$ $\sigma\left(A_{\text {l, },(3)}\right)$ Similarly to (106), we define bounded nonnegative measurable functions

$$
0_{(1) \tau, n}^{ \pm}(x)=\max \left\{ \pm\left(\frac{1}{\varrho_{\left.g,()^{(n)}\right)}(x)}-\frac{1}{\varrho_{\tau}(x)}\right), 0\right\},
$$

and corresponding self-adjoint operators $\Theta_{n}^{ \pm}(\omega, \tau)$ defined as in (107), so we get analogous inequalities to $(108)-(110)$ We have $\lim _{n \rightarrow \infty}\left\langle\psi, \Theta_{n}^{ \pm}(\omega, \tau) \psi\right\rangle=0$ for any $\psi \in \mathscr{L}$, since

$$
\left|\varrho_{\left.g,()^{(n)}\right)}(x)-\varrho_{\tau}(x)\right| \%_{0,\left(/_{n}-4 l_{u^{\prime \prime}}\right)}(x) \leqq\left(\ell_{n}+1\right)^{-(d+1)} \varrho_{0,+} U_{+} \%_{0,\left(/_{n}-4 l_{n}\right)}(x)
$$

As before, this last observation allows us to apply Lemma 45 and conclude that

$$
\sigma(A(\tau)) \subset \overline{\bigcap_{n=0}^{\infty} \sigma\left(A_{g,()^{(n)}}\right)}=\sigma\left(A_{\left(y_{1(1)}\right.}\right),
$$

which implies the validity of (114) 
Given a real number $h,|h|<\frac{1}{U_{+}^{\prime}}$, let

$$
A(h)=A\left(\underline{Q}_{h}\right) \quad \text { with } \underline{Q}_{h}(x)=\varrho_{0}(x)[1+h U(x)]
$$

If $|h| \leqq y$, and we define $\tau(h) \in \mathscr{T}_{g}$ by $\tau(h)_{l}=h$ for all $i \in \mathbb{Z}^{d}$, we have $\underline{Q}_{h}=\varrho_{\tau(h)}$ and $A(h)=A(\tau(h))$

Lemma 20. Let $A(h),|h|<\frac{1}{l^{\prime}}$, be given by (119), with $\varrho_{0}$ and $U$ given in Assumption 1 Let $\Lambda=\Lambda,\left(x_{0}\right)$ for some $x_{0} \in \mathbb{R}^{d}$ and $\ell \succeq q$ The positive selfadjoint operator $\left(A(\stackrel{\circ}{h})_{1}\right.$ has compact resolvent, so let $0<\mu_{1}(h) \leqq \mu_{2}(h) \leqq$ be its eigenvalues, repeated according to their (finite) multiplicity. Then each $\mu_{l}(h)$, $j=1,2, \quad$, is a Lipschitz continuous, strictly decreasing function of $h$, with

$$
\delta_{-}(g) \max _{l=1,2}\left\{\mu_{l}\left(h_{l}\right)\right\} \leqq \frac{\mu_{j}\left(h_{1}\right)-\mu_{l}\left(h_{2}\right)}{h_{2}-h_{1}} \leqq \delta_{+}(g) \min _{l=1,2}\left\{\mu_{j}\left(h_{l}\right)\right\}
$$

for anv $h_{1}, h_{2} \in(-g, g), 0<g<\frac{1}{L^{\prime}}$, where $\delta_{ \pm}(g)$ are given in (17)

Proof Let $h_{1}, h_{2} \in(-g, g), 0<g<\frac{1}{U}$, it follows that

$$
\frac{1}{\varrho_{h_{1}}(x)}-\frac{1}{\varrho_{h_{2}}(x)}=\frac{\left(h_{2}-h_{1}\right) \varrho_{0}(x) U(x)}{\varrho_{h_{1}}(x) \varrho_{h_{2}}(x)},
$$

So

$$
\frac{1+\delta_{-}(g)\left(h_{2}-h_{1}\right)}{\varrho_{h_{2}}(x)} \leqq \frac{1}{\varrho_{h_{1}}(x)} \leqq \frac{1+\delta_{+}(g)\left(h_{2}-h_{1}\right)}{\varrho_{h_{2}}(x)},
$$

and

$$
\frac{1-\delta_{+}(g)\left(h_{2}-h_{1}\right)}{\varrho_{h_{1}}(x)} \leqq \frac{1}{Q_{h_{2}}(x)} \leqq \frac{1-\delta_{-}(g)\left(h_{2}-h_{1}\right)}{\varrho_{h_{1}}(x)}
$$

From (122) we get

$$
\left(1+\delta_{-}(g)\left(h_{2}-h_{1}\right)\right) A\left(\stackrel{\circ}{h}_{2}\right)_{1} \leqq A\left(\stackrel{\circ}{h}_{1}\right)_{1} \leqq\left(1+\delta_{+}(g)\left(h_{2}-h_{1}\right)\right) A\left(\stackrel{\circ}{h_{2}}\right)_{1},
$$

so it follows from the min-max principle that, for any $j=1,2$,

$$
\left(1+\delta_{-}(g)\left(h_{2}-h_{1}\right)\right) \mu_{j}\left(h_{2}\right) \leqq \mu_{j}\left(h_{1}\right) \leqq\left(1+\delta_{+}(g)\left(h_{2}-h_{1}\right)\right) \mu_{j}\left(h_{2}\right),
$$
i e,

$$
\delta_{-}(g)\left(h_{2}-h_{1}\right) \leqq \frac{\mu_{j}\left(h_{1}\right)-\mu_{j}\left(h_{2}\right)}{\mu_{j}\left(h_{2}\right)} \leqq \delta_{+}(g)\left(h_{2}-h_{1}\right)
$$

Similarly, using (123) we get

$$
\delta_{-}(g)\left(h_{2}-h_{1}\right) \leqq \frac{\mu_{j}\left(h_{1}\right)-\mu_{j}\left(h_{2}\right)}{\mu_{j}\left(h_{1}\right)} \leqq \delta_{+}(g)\left(h_{2}-h_{1}\right)
$$

Equation (120) follows from (126) and (127) The properties of the functions $\mu_{j}(h)$ follows

The following corollary follows immediately from Theorem 14, Lemmas 19 and 20 , and the min-max principle 
Corollary 21. Let the random operator $A_{g}$ defined by' (18) satisfy Assumption 1, and let $\left\{\ell_{n}, n=0,1,2, \ldots\right\}$ be a sequence in $\mathbb{N}$ such that $t_{0}=q$ and $\ell_{n} \prec \ell_{n+1}$ for each $n=0,1,2$, Then

$$
\Sigma_{g}=\overline{\bigcup_{h \in[-g, g]} \sigma(A(h))}=\overline{\bigcup_{h \in[-g . g] n \geqq 1} \sigma\left(A(\stackrel{\circ}{h})_{1_{\iota_{n}}(0)}\right)}
$$

In particular, $\Sigma_{y}$ is increasing in $y$

42 Inside the gap We now prove Theorem 3. As $\Sigma_{g}$ is increasing in $g$, we expect the gap to shrink as we increase $g$ until it either disappears at some $g_{0}$, or it remains open for all allowed $g$ Thus we define

$$
y_{0}=\sup \left\{g \in\left[0, \frac{1}{U_{+}}\right), \quad \Sigma_{g} \cap(a, b) \neq(a, b)\right\}
$$

Let $\left\{\ell_{n}, n=0,1,2, \quad\right\}$ be as in Corollary $21, h \in[-g, g]$, and let $0<\mu_{1}^{(n)}(h)$ $\leqq \mu_{2}^{(n)}(h) \leqq$ be the eigenvalues of $A(\stackrel{\circ}{h})_{1_{n}}, \Lambda_{n}=\Lambda_{\ell_{n}}(0)$, repeated according to their (finite) multiplicity, notice $\lim _{, \rightarrow \infty} \mu_{1}^{(n)}(h)=\infty$ By Lemma 20 each $\mu_{1}^{(n)}(h)$ is a strictly decreasing continuous function of $h$, hence it follows from Corollary 21 that

$$
\Sigma_{y}=\overline{\bigcup_{n \geqq 1} \bigcup_{h \in[-g, g]} \sigma\left(\AA(h)_{t_{n}}\right)}=\overline{\bigcup_{n \geqq 1} \bigcup_{j=0}^{\infty}\left[\mu_{j}^{(n)}(g), \mu_{j}^{(n)}(-g)\right]}
$$

In particular, $\Sigma_{y}$ is a countable union of disjoint closed intervals, none contained in the original gap $(a, b)$, so for $g<g_{0}$ we can define $a(g)$ and $b(g)$ by (20) Since $\Sigma_{g}$ is increasing in $g \in\left[0, \frac{1}{U_{+}}\right)$by Corollary 21 , it follows that $a(g)$ and $-b(g)$ are increasing functions in $\left[0, g_{0}\right)$

For each $n$ let

$$
j_{n}=\max \left\{j, \mu_{j}^{(n)}(0) \leqq a\right\},
$$

so using Assumption 2 and (56) in Theorem 14, we have

$$
j_{n}+1=\min \left\{j, \mu_{j}^{(n)}(0) \geqq b\right\}
$$

If $g<y_{0}$, it follows from the definition of $j_{n}$, Assumption 2 and (56)-(57) in Theorem 14 , that $\mu_{\mu_{n}}(-g)$ and $-\mu_{n_{n}+1}(g)$ are both increasing in $n$, and

$$
\begin{aligned}
& a(g)=\lim _{n \rightarrow \infty} \mu_{j_{n}}(-g), \\
& b(g)=\lim _{n \rightarrow \infty} \mu_{j_{n}+1}(g)
\end{aligned}
$$

Thus, given $0 \leqq g_{1}<g_{2}<g_{0}$, we can conclude from (120) that

$$
\begin{aligned}
& \delta_{-}\left(g_{2}\right) a\left(g_{2}\right) \leqq \frac{a\left(g_{2}\right)-a\left(g_{1}\right)}{g_{2}-g_{1}} \leqq \delta_{+}\left(g_{2}\right) a\left(g_{1}\right), \\
& \delta_{-}\left(g_{2}\right) b\left(g_{1}\right) \leqq \frac{b\left(g_{1}\right)-b\left(g_{2}\right)}{g_{2}-g_{1}} \leqq \delta_{+}\left(g_{2}\right) b\left(g_{2}\right)
\end{aligned}
$$


The Lipschitz continuity of $a(g)$ and $b(g)$ follows, as well as

$$
\int_{y_{1}}^{y_{2}} \delta_{-}(h) d h \leqq \log \frac{a\left(g_{2}\right)}{a\left(g_{1}\right)}, \quad \log \frac{b\left(g_{1}\right)}{b\left(g_{2}\right)} \leqq \int_{y_{1}}^{g_{2}} \delta_{+}(h) d h,
$$

so we obtain (21) and (22), from which we get (19)

If $g_{0}<\frac{1}{l^{\prime}}$, we must have $\lim _{g \uparrow g_{0}} a(g)=\lim _{g \uparrow g_{0}} b(g)$ This follows from (130), (133) and (134), since by $(120)$ each $\mu_{1}^{(n)}(h)$ is a Lipschitz continuous function of $h \in\left(-\frac{1}{L_{-}^{\prime}}, \frac{1}{L^{\prime}}\right)$, uniformly in $n$ Thus, if $y \in\left[g_{0}, \frac{1}{L^{\prime}}\right)$ it follows that $[\hat{a}, \hat{b}] \subset \Sigma_{y}$, we set $a(g)=h(g)=\lim _{g \uparrow g_{0}} a(g)$

Theorem 3 is proven

\section{A Wegner-Type Estimate}

Given an open cube $\Lambda$ in $\mathbb{R}^{d}$, we will denote by $A_{y_{1} \Lambda}=A_{y_{1}(1), 1}$ the restriction of $A_{\left(\text {(.) }^{\prime}\right)}$ to $\Lambda$ with Dirichlet boundary condition (e g., [RS4]) Notice that each $A_{\left(y^{(1)}\right)} 1$ is a nonnegative self-adjoint operator on $L^{2}(\Lambda)$ with compact resolvent, and measurability follows from Theorem 38 We can thus define $n_{g}{ }_{1}(E)=n_{g_{\text {. }()_{1}{ }_{1}}(E)}$ as the number of eigenvalues of $A_{\text {g.(1). } 1}$ less than $E$, clearly $n_{\text {g, (1), } 1}(E)=0$ for $E \leqq 0$ Notice that $n_{g,(1), 1}(E)$ is the distribution function of the measure $n_{g,(1), 1}(d E)$ given by

$$
\int h(E) n_{\text {g. (1), } 1}(d E)=\operatorname{Tr}\left(h\left(A_{\text {g, (1), } 1}\right)\right)
$$

for positive continuous functions $h$ of a real variable

We will say that the random operator $A_{y}$ defined by (18) satisfies Assumption 1', if it satisfies all of Assumption 1 with the exception of the requirement that $\varrho_{0}(r)$ be a $q$-periodic function.

We have the following "a priori" estimate

Lemma 22. Let the random operator $A_{g}$ defined by (18) satisfy Assumption $1^{\prime}$ There evists a constant $C_{1}=C_{1}\left(d, Q_{0}+\right)<\infty$ such that we have

$$
n_{g(1) . \Lambda}(E) \leqq C_{1}|\Lambda| E^{\frac{d}{2}}
$$

for all (1) $\in[-1,1]^{\mathbb{Z}^{\prime}}$, for all $E \geqq 0$ and all cubes $\Lambda$ in $\mathbb{Z}^{d}$

Proof Let $\Delta_{1}$ be the Laplacian on $\Lambda$ with Dirichlet boundary condition We have

$$
A_{\text {g, (1), }, 1} \geqq-\frac{1}{\varrho_{0+}\left(1+g U_{+}\right)} \Delta_{1} \geqq-\frac{1}{2 \varrho_{0+}} \Delta_{1} .
$$

Thus (139) follows from [RS4, p 267 (118)]

Theorem 23 (Wegner-type estimate). Let the random operator $A_{y}$ defined by (18) satisfy Assumption 1' There exists a constant $Q<\infty$, depending only on the dimension $d$ and the constants $r_{u}$ and $Q_{0,+}$, such that

$$
\mathbb{P}\left\{\operatorname{dist}\left(\sigma\left(A_{g,(1), \Lambda}\right), E\right) \leqq \eta\right\} \leqq Q \frac{U_{-}+2 U_{+}}{g U_{+}\left(1-g U_{+}\right) U_{-}}\|\rho\|_{\infty}|E|^{\frac{d}{2}-1} \eta|\Lambda|^{2}
$$

for all $E>0$, cubes $\Lambda$ in $\mathbb{R}^{d}$, and all $\eta \in[0, E]$ 
Proof Let us pick $\kappa \in\left(1, \frac{1}{g U_{+}}\right)$, say $\kappa=\frac{1+g U_{+}}{2 g U^{\prime}}$, we write

$$
\hat{\gamma}_{g(1)}=\hat{\imath}+g \sum_{i \in \mathbb{Z}^{\prime}} s_{l} u_{l}
$$

where

$$
\hat{\imath}=1-\kappa g \sum_{l \in \mathbb{Z}^{\prime}} u_{l}>\frac{1-g U_{+}}{2}>0,
$$

and $s_{i}=\left(\omega_{1}+\kappa \in[\kappa-1, k+1]\right.$ for each $i \in \mathbb{Z}^{d}$

Now let $f$ be an arbitrary continuous function on the real line with compact support As in [FK2, Lemma 7], we have

$$
\begin{aligned}
& -\frac{\partial}{\partial s_{i}} \int n_{(1,(), 1}(E) f(E) d E=\operatorname{Tr}\left\{\left(-\frac{\hat{c}}{\partial s_{i}} A_{(y,(), A}\right) f\left(A_{q,(1), 1}\right)\right\} \\
& =\operatorname{Tr}\left\{A_{g,(1), A}\left(\frac{g u_{i}}{\varrho_{g,(1)} \hat{\gamma}_{g,(1)}}\right) f\left(A_{g,(1), 1}\right)\right\} \text {, }
\end{aligned}
$$

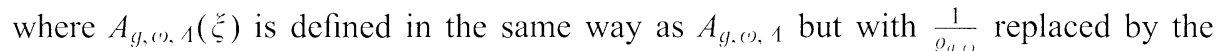
function

Thus,

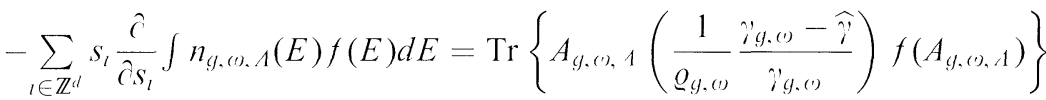

$$
\begin{aligned}
& =\operatorname{Tr}\left\{A_{g,(1) 1} f\left(A_{(y,(), 1}\right)\right\}-\operatorname{Tr}\left\{A_{(g,(), 1}\left(\frac{1}{\underline{Q}_{g,(1)}} \frac{\widehat{\gamma}}{\hat{\gamma}_{g,(1)}}\right) f\left(A_{g,(1), A}\right)\right\}
\end{aligned}
$$

But

$$
\frac{\hat{i} g()}{\hat{\imath}}=\frac{\hat{i}+g \sum_{i \in \mathbb{Z}^{l}} s_{l} u_{l}}{\hat{\imath}} \geqq 1+\frac{(\kappa-1) g U_{-}}{1-\kappa g U_{-}} \geqq 1+\frac{\left(1-g U_{+}\right) U_{-}}{2 U_{+}},
$$

and $f \geqq 0$, so

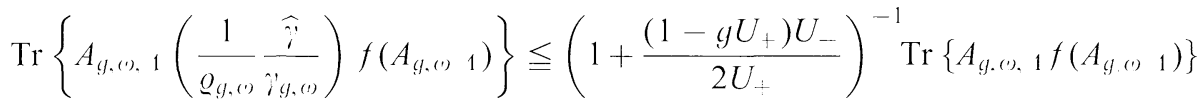

We conclude that

$$
\begin{aligned}
\operatorname{Tr} & \left\{A_{(y,(1), 1} f\left(A_{(y,(), A}\right)\right\} \\
& \leqq\left(1+\frac{2 U_{+}}{\left(1-g U_{+}\right) U_{-}}\right)\left(-\sum_{i \in \mathbb{Z}^{d}} s_{l} \frac{\hat{c}}{\partial s_{l}} \int n_{g,(1), A}(E) f(E) d E\right)
\end{aligned}
$$


For given $j \in \mathbb{Z}^{d}$ let $\omega^{(/)}=\left\{\omega_{l}, i \in \mathbb{Z}^{d} \backslash\{j\}\right\}$, and denote the corresponding expectation by $\mathbb{E}^{(/)}$We have

$$
\begin{aligned}
& \mathbb{E}\left(-\frac{\partial}{\partial s_{j}} \int n_{g,(1), \Lambda}(E) f(E) d E\right) \\
& =\mathbb{E}^{(j)}\left(\int_{n-1}^{n+1}\left[-\frac{\partial}{\partial s_{l}} \int n_{g,(j) A}(E) f(E) d E\right] \rho\left(s_{l}-\kappa\right) d s_{j}\right)
\end{aligned}
$$

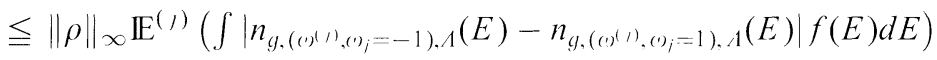

$$
\begin{aligned}
& \leqq \delta_{1.1}\|\rho\|_{\infty} 2 C_{1}|\Lambda| \int E^{\frac{d}{2}} f(E) d E \text {, }
\end{aligned}
$$

where we used (139), here $\delta_{j . \Lambda}=0$ if $u_{\jmath} \equiv 0$ in $\Lambda$ and $\delta_{\text {J.A }}=1$ otherwise Since the function $u$ has support in a cube of side $2 r_{u}$, there exists a constant $C_{2}<\infty$, depending only on $r_{u}$ and the dimension $d$, such that

$$
\sum_{i \in \mathbb{Z}^{d}} \delta_{i, \Lambda} \leqq C_{2}|\Lambda|
$$

for all cubes $\Lambda$ in $\mathbb{Z}^{d}$

Let $\hat{n}_{g ., 1}(d E)=\mathbb{E}\left(n_{g,(\omega . \Lambda}(d E)\right)$ For functions $f$ as above, it now follows from (144),(145) and (146) that

$$
\int E f(E) \hat{n}_{g 1}(d E) \leqq 2 C_{1} C_{2}(\kappa+1)\left(1+\frac{2 U_{+}}{\left(1-g U_{+}\right) U_{--}}\right)\|\rho\|_{\infty}|\Lambda|^{2} \int E^{\frac{d}{2}} f(E) d E
$$

It follows that $\hat{n}_{y, \Lambda}(d E)$ is absolutely continuous with

$$
\frac{\hat{n}_{l, \Lambda}(d E)}{d E} \leqq C_{3}|\Lambda|^{2} E^{\frac{d}{2}-1} \quad \text { for } E \geqq 0
$$

where

$$
C_{3}=4 C_{1} C_{2} \frac{U_{-}+2 U_{+}}{g U_{+}\left(1-g U_{+}\right) U_{-}}\|\rho\|_{\infty}
$$

The estimate (141) now follows by a standard argument:

$$
\begin{aligned}
\mathbb{P}\{\operatorname{dist} & \left.\left(\sigma\left(A_{g,(1), 1}\right), E\right)<\eta\right\} \leqq \mathbb{P}\left\{\int_{[E-\eta) \cdot E+\eta]} n_{g,(1), A}(d E) \geqq 1\right\} \\
\leqq & \int_{[E-\eta, E+\eta]} \hat{n}_{g, A}(d E) \leqq 2^{\frac{d}{2}} C_{3} E^{\frac{d}{2}-1} \eta|\Lambda|^{2}
\end{aligned}
$$

for all $E>0$ and $0 \leqq \eta \leqq E$. 


\section{Localization}

In this section we prove Theorems 6 and 7 To do so we develop a multiscale analysis appropriate for random perturbations of periodic operators on $\mathbb{R}^{d}$, based on the von Dreifus-Klein [DK] multiscale analysis We will work with random operators $A_{g}$ of the form given in (18), but our method works also for random Schrödinger operators

Let the operator $A$ be as in (10) with (11) Given an open cube $\Lambda$ in $\mathbb{R}^{d}$, we will denote by $A_{1}$ the restriction of $A$ to $A$ with Dirichlet boundary condition (e g, [RS4]) Each $A_{1}$ is a nonnegative self-adjoint operator on $L^{2}(\Lambda)$ with compact resolvent $R_{1}(z)=\left(A_{1}-z\right)^{-1}$ If $\Lambda=\Lambda_{L}(x)$, we will write $A_{r, L}=A_{\Lambda_{I}(r)}$ and $R_{\Upsilon, L}(z)$ $=R_{A_{l}(x)}(z)$ The norm in $L^{2}\left(\Lambda_{L}(x)\right)$ and also the corresponding operator norm will both be denoted by \|\|$_{r, L}$. If $\Lambda_{1} \subset \Lambda_{2}$ are open cubes, $J_{\Lambda_{1}}^{\Lambda_{2}} L^{2}\left(\Lambda_{1}\right) \rightarrow L^{2}\left(\Lambda_{2}\right)$ is the canonical injection. If $\Lambda_{l}=\Lambda_{L_{l}}\left(x_{i}\right), i=1,2$, we write \|\|$_{r_{1}, L_{1}}^{x_{2} \cdot L_{2}}$ for the (operator) norm in $\mathscr{B}\left(L^{2}\left(\Lambda_{L_{1}}\left(x_{1}\right)\right), L^{2}\left(\Lambda_{L_{2}}\left(x_{2}\right)\right)\right)$ and $J_{r_{1}, L_{1}}^{r_{2}, L_{2}}=J_{\Lambda_{L_{1}}\left(x_{1}\right)}^{\Lambda_{L_{2}}\left(r_{2}\right)}$. If $\varphi \in L^{\infty}(\Lambda)$, we also denote the operator multiplication by $\varphi$ on $L^{2}(\Lambda)$ by $\varphi$.

61 The basic technical tools The basic tool to relate resolvents in different scales is a local resolvent identity (LRI), which was used in a similar context by Combes and Hislop $[\mathrm{CH}]$.

Lemma 24 (LRI). Let $\Lambda_{1} \subset \Lambda_{2}$ be open cubes in $\mathbb{R}^{d}$, let $H_{i}$ be a self-adjoint operator on $L^{2}\left(\Lambda_{i}\right), i=1,2$, with $R_{l}(z)=\left(H_{l}-z\right)^{-1}$, and let $\varphi_{1} \in L^{\infty}\left(\Lambda_{1}\right)$ Then, for any $z \notin \sigma\left(H_{1}\right) \cup \sigma\left(H_{2}\right)$ we have

$$
R_{2}(z) J_{1_{1}}^{A_{2}} \varphi_{1}=J_{A_{1}}^{A_{2}} \varphi_{1} R_{1}(z)+R_{2}(z)\left(J_{A_{1}}^{A_{2}} \varphi_{1} H_{1}-H_{2} J_{\Lambda_{1}}^{A_{2}} \varphi_{1}\right) R_{1}(z)
$$

as quadratic forms on $L^{2}\left(\Lambda_{2}\right) \times L^{2}\left(\Lambda_{1}\right)$

Proof We clearly have

$$
R_{2}(z) J_{1_{1}}^{\lambda_{1}} \varphi_{1} \psi_{1}=R_{2}(z) J_{\Lambda_{1}}^{A_{2}} \varphi_{1}\left(H_{1}-z\right) R_{1}(z) \psi_{1} \quad \text { for all } \psi_{1} \in L^{2}\left(\Lambda_{1}\right),
$$

and

$$
\left\langle\psi_{2}, J_{1_{1}}^{1_{2}} \varphi_{1} R_{1}(z) \psi_{1}\right\rangle_{L^{2}\left(A_{2}\right)}=\left\langle\left(H_{2}-z^{*}\right) R_{2}\left(z^{*}\right) \psi_{2}, J_{1_{1}}^{1_{2}} \varphi_{1} R_{1}(z) \psi_{1}\right\rangle_{L^{2}\left(A_{2}\right)}
$$

for all $\psi_{1} \in L^{2}\left(\Lambda_{1}\right), \psi_{2} \in L^{2}\left(\Lambda_{2}\right)$, so (151) follows.

For operators of the form given by (10), the LRI yields the smooth resolvent identity (SRI)

Lemma 25 (SRI). Let the operator $A$ be given by (10) with (11), let $\Lambda_{1} \subset \Lambda_{2}$ be open cubes in $\mathbb{R}^{d}$, and let $\varphi_{1} \in C_{0}^{1}\left(\Lambda_{1}\right)$ Then, for any $z \notin \sigma\left(A_{\Lambda_{1}}\right) \cup \sigma\left(A_{A_{2}}\right)$ we have

$$
\begin{aligned}
R_{A_{2}}(z) J_{A_{1}}^{\lambda_{2}} \varphi_{1} & =J_{A_{1}}^{A_{2}} \varphi_{1} R_{A_{1}}(z) \\
& +R_{A_{2}}(z)\left(-J_{A_{1}}^{A_{1}} \frac{1}{\varrho}\left(\nabla \varphi_{1}\right) \cdot \nabla+\nabla \cdot J_{1_{1}}^{A_{2}}\left(\nabla \varphi_{1}\right) \frac{1}{\varrho}\right) R_{A_{1}}(z)
\end{aligned}
$$

as quadratic forms on $L^{2}\left(\Lambda_{2}\right) \times L^{2}\left(\Lambda_{1}\right)$ 
Proof Using (151), (10), and the definition of Dirichlet boundary condition we get (154)

The other basic tool for the multiscale analysis is a Simon-Lieb-type inequality (SLI), which we need to adapt to the continuum It is used to obtain decay in a larger scale from decay in a given scale

Since we are working in a background medium of period $q \in \mathbb{N}$ (see Assumption 1), we will work with boxes $\Lambda_{L}(x)$ with $x \in q \mathbb{Z}^{d}$ and $L \in 2 q \mathbb{N}$, so the background will be the same in all boxes in a given scale $L$ For such boxes (with $L \geqq 4 q$ ) we set

$$
\Upsilon_{L}(x)=\left\{y \in q \mathbb{Z}^{d}, \quad\|y-x\|=\frac{L}{2}-q\right\}
$$

and

$$
\tilde{\Upsilon}_{L}(x)=\Lambda_{L-q}(x) \backslash \overline{\Lambda_{L-3 q}(x)}, \quad \hat{\Upsilon}_{L}(x)=\Lambda_{L-\frac{3 q}{2}}(x) \backslash \overline{\Lambda_{L-\frac{S_{q}}{2}}(x)}
$$

We also set

$$
\chi_{1}=\psi_{1, q} \quad \text { and } \quad \Gamma_{1 L}=\psi_{\dot{r}_{l}(1)}, \quad \hat{\Gamma}_{1, L}=\psi_{\hat{r}_{l}(r)}
$$

Notice

$$
\Gamma_{r, L}=\sum_{v \in Y_{l}(r)} \%_{v} \text { a e }
$$

and

$$
\left|\Upsilon_{L}(x)\right| \leqq d(L-2 q+1)^{d-1}
$$

In addition each $\Lambda_{L}(x)$ will be equipped with a function $\Phi_{r, L}$ constructed in the following way. we fix an even function $\xi \in C_{0}^{1}(\mathbb{R})$ with $0 \leqq \xi(t) \leqq 1$ for all $t \in \mathbb{R}$, such that $\xi(t)=1$ for $|t| \leqq \frac{q}{4}, \xi(t)=0$ for $|t| \geqq \frac{3 q}{4}$, and $\left|\xi^{\prime}(t)\right| \leqq \frac{3}{q}$ for all $t \in \mathbb{R}$ (Such a function always exists) We define

$$
\xi_{L}(t)= \begin{cases}1, & \text { if }|t| \leqq \frac{L}{2}-\frac{5 q}{4} \\ \xi\left(|t|-\left(\frac{L}{2}-\frac{3 q}{2}\right)\right), & \text { if }|t| \geqq\left(\frac{L}{2}-\frac{3 q}{2}\right)\end{cases}
$$

and set

$$
\Phi_{\mathrm{r}, L}(y)=\Phi_{L}(y-x) \quad \text { for } y \in \mathbb{R}^{d}, \quad \text { with } \Phi_{L}(y)=\prod_{l=1}^{d} \check{\zeta}_{L}\left(y_{i}\right)
$$

We have $\Phi_{\uparrow, L} \in C_{0}^{1}\left(\Lambda_{L}(x)\right), 0 \leqq \Phi_{r, L} \leqq 1$,

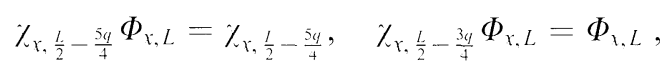

and

$$
\hat{\Gamma}_{\curlyvee, L} \nabla \Phi_{\curlyvee, L}=\nabla \Phi_{\curlyvee, L}, \quad\left|\nabla \Phi_{\curlyvee, L}\right| \leqq \frac{3 \sqrt{d}}{q}
$$


Lemma 26 (SLI). Let the operator $A$ be given by (10) with (11). Then for any $\ell, L \in 2 q \mathbb{N}$ with $4 q \leqq \ell<L-3 q, x, y \in q \mathbb{Z}^{d}$ with $2\|y-x\| \leqq L-\ell-3 q$ (so $\left.\Lambda_{f}(y) \subset \Lambda_{L-3 y}(x)\right)$, and $z \notin \sigma\left(A_{r, L}\right) \cup \sigma\left(A_{1, \ell}\right)$, we have

$$
\left\|\Gamma_{r, L} R_{r, L}(z) \psi_{v}\right\|_{r, L} \leqq \gamma_{z} \ell^{d-1}\left\|\Gamma_{v, /} R_{v, /}(z) \psi_{v}\right\|_{v, /}\left\|\Gamma_{r, L} R_{x, L}(z) \chi_{v^{\prime}}\right\|_{r, L}
$$

for some $y^{\prime} \in \Upsilon_{1 . /}$, with

$$
\gamma_{z}=\frac{6 d^{\frac{3}{2}}}{q \varrho-} \Theta_{\frac{4}{4}}(1+|z|),
$$

where $\Theta_{\frac{q}{4}}=\Theta\left(d, \varrho_{ \pm}, \frac{q}{4}\right)$ is the constant given in Proposition 39

Proof Using (162), (154) and $\Gamma_{\uparrow, L} J_{1, L}^{r, L} \Phi_{1, \ell}=0$ we obtain

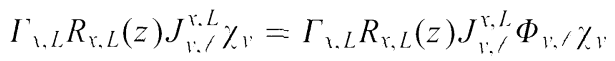

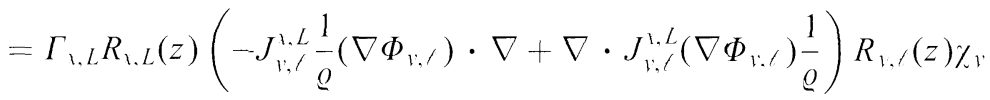

as quadratic forms on $L^{2}\left(\Lambda_{L}(x)\right) \times L^{2}\left(\Lambda_{\lambda}(y)\right)$

We now use (163) and (11) to get

$$
\begin{aligned}
& \left\|\Gamma_{\imath, L} R_{r, L}(z) J_{1, \ell}^{r, L} \frac{1}{\varrho}\left(\nabla \Phi_{v, f}\right) \cdot \nabla R_{v, /}(z) \psi_{v}\right\|_{1, \ell}^{r, L}
\end{aligned}
$$

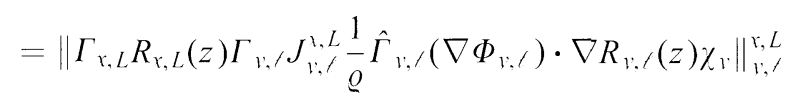

$$
\begin{aligned}
& \leqq \frac{3 \sqrt{d}}{q \varrho-}\left\|\hat{\Gamma}_{1: /} \nabla R_{v, /}(z) \psi_{1} \cdot\right\|_{1, \ell}\left\|\Gamma_{r, L} R_{r, L}(z) \Gamma_{1, r}\right\|_{r, L}
\end{aligned}
$$

and

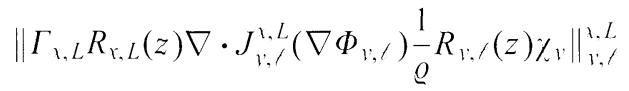

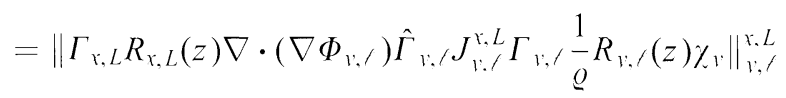

$$
\begin{aligned}
& \leqq \frac{1}{\varrho_{-}}\left\|\Gamma_{v, \ell} R_{v, \ell}(z) \psi_{v}\right\|_{1,, \ell}\left\|\Gamma_{r, L} R_{\mathrm{r}, L}(z) \nabla \cdot\left(\nabla \Phi_{v, \ell}\right) \hat{\Gamma}_{i, \ell}\right\|_{\mathrm{i} . L} \\
& \leqq \frac{3 \sqrt{d}}{q \varrho \ldots}\left\|\Gamma_{1, \ell}, R_{1, /}(z) \%_{1}\right\|_{1,, /}\left\|\hat{\Gamma}_{v, /} \nabla R_{\mathrm{r}, L}\left(z^{*}\right) \Gamma_{r, L}\right\|_{r, L}
\end{aligned}
$$

Appealing to Proposition 39 we obtain

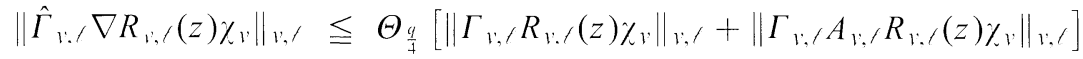

$$
\begin{aligned}
& \leqq \Theta_{\frac{q}{4}}(1+|z|)\left\|\Gamma_{v, /} R_{v, /}(z) \psi_{v}\right\|_{1, /}
\end{aligned}
$$


and

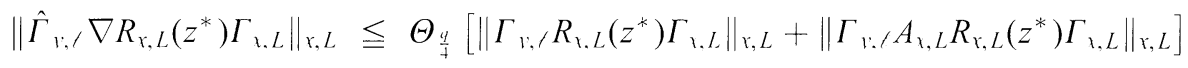

$$
\begin{aligned}
& \leqq \Theta_{\frac{y}{4}}(1+|z|)\left\|\Gamma_{\mathrm{r}, f} R_{\mathrm{r}, L}\left(z^{*}\right) \Gamma_{\mathrm{r}, L}\right\|_{\mathrm{r}, L} \\
& =\Theta_{\frac{q}{4}}(1+|z|)\left\|\Gamma_{\curlyvee, L} R_{\curlyvee, L}(z) \Gamma_{1, /}\right\|_{\Upsilon, L}
\end{aligned}
$$

Thus,

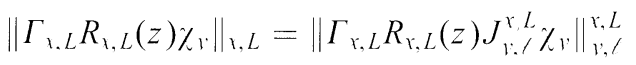

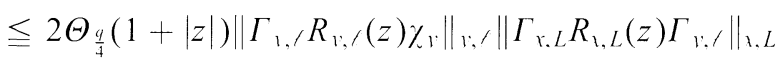

Hence (164) follows from (175),(158) and (159)

The last tool in this subsection is the eigenfunction decay' inequality (EDI), an inequality that gives decay for generalized eigenfunctions (see Appendix B 2 ) from decay of local resolvents

Lemma 27 (EDI). Let the operator $A$ be given by (10) with (11), and let $\psi$ be a generalized eigenfunction for a given $z \in \mathbb{C}$ For any $x \in q \mathbb{Z}^{d}$ and $t \in 2 q \mathbb{N}$ with $1 \geqq 4 q$, such that $z \notin \sigma\left(A_{\downarrow}, 1\right)$, we have

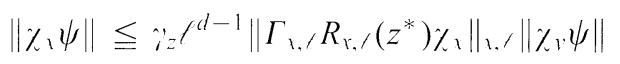

for some $y \in \Upsilon_{1.1}$, with $i_{z}$ as in (165)

Proof. Since $(A-z I) \psi=0$ weakly (see (24l)), we also have $\Phi_{\text {r. } /}(A-z I) \psi=0$ weakly, so $J^{r / t} \Phi_{\curlywedge . /}(A-z I) \psi=0$ weakly in $L^{2}(\Lambda /(x))$, where $J^{1,}=J_{\mathbb{R}^{\prime l}}^{A^{\prime \prime(1)}}$. Thus

$$
\begin{aligned}
& J^{r, /} \psi_{r} \psi=\varkappa_{\curlywedge} R_{\curlyvee, /}(z)\left(A_{\curlyvee, /}-z I\right) J^{r, /} \Phi_{r, /} \psi \\
& =\varkappa_{\curlywedge} R_{\curlywedge, /}(z)\left(A_{\uparrow, /} J^{\uparrow /} \Phi_{\curlywedge, /}-J^{\curlywedge /} \Phi_{\curlywedge, /} A\right) \psi \\
& =\chi_{\imath} R_{r, /}(z)\left(-\nabla \cdot J^{1, \gamma} \frac{1}{\varrho}\left(\nabla \Phi_{r, /}\right)+J^{\curlywedge, /}\left(\nabla \Phi_{r, /}\right) \cdot \frac{1}{\varrho} \nabla\right) \psi
\end{aligned}
$$

weakly in $L^{2}(\Lambda,(x))$

Proceeding as in the proof of Lemma 26, we have

$$
\begin{aligned}
& \left\|{ }_{r} R_{r, \prime}(z) \nabla \cdot J^{1, \prime} \frac{1}{\varrho}\left(\nabla \Phi_{r},\right) \psi\right\|_{r, /}
\end{aligned}
$$

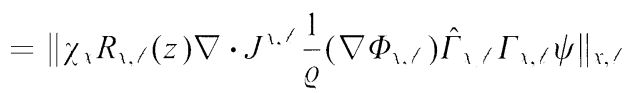

$$
\begin{aligned}
& \leqq \frac{1}{\varrho_{-}}\left\|\hat{\Gamma}_{\mathrm{r}, /}\left(\nabla \Phi_{\mathrm{\imath}, 1}\right) \cdot \nabla R_{\mathrm{r}, /}\left(z^{*}\right) \psi_{r}\right\|_{\mathrm{r}, \mathrm{t}}\left\|\Gamma_{\mathrm{r}, /} \psi\right\|
\end{aligned}
$$

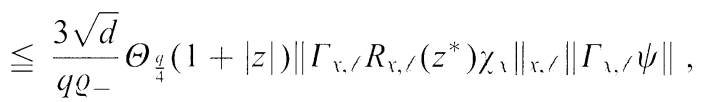


and

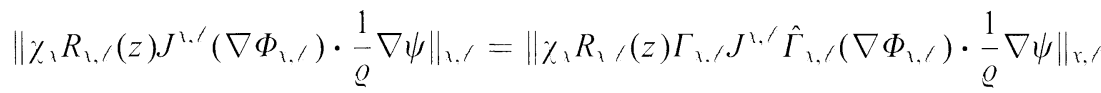

$$
\begin{aligned}
& \leqq \frac{3 \sqrt{d}}{q \varrho-} \Theta_{\frac{q}{4}}(1+|z|)\left\|\Gamma_{\imath, /} R_{\curlywedge,},\left(z^{*}\right) \psi_{,}\right\|_{\imath, /}\left\|I_{1, /} \psi\right\|
\end{aligned}
$$

Thus (176) follows

62 The multiscale analysis Motivated by the SLI (164) and the EDI (176) we make the following deterministic definition (see $[\mathrm{CH}]$ for a similar definition)

Definition 28. Let the operator $A$ be as in (10) with (11) Given $m>0, E>0$, $x \in q \mathbb{Z}^{d}$ and $L \in 2 q \mathbb{N}, L \geqq 4 q$, we sav, that the cube $\Lambda_{L}(x)$ is $(m, E)$-regular, if $E \notin \sigma\left(A_{\text {เ.L. }}\right)$ and

$$
\left\|\Gamma_{1, L} R_{1, L}(E) \psi_{1}\right\|_{1, L} \leqq \mathrm{e}^{-m \frac{L}{2}}
$$

We say that $\psi \in L^{2}\left(\mathbb{R}^{d}\right)$ decays exponentially fast with mass $m>0$ if

$$
\limsup _{\|x\| \rightarrow \infty} \frac{\log |\psi(x)|}{\|x\|} \leqq-m
$$

Motivated by the EDI, which is formulated for $\left\|\psi_{r} \psi\right\|$, we say that $\psi$ decays $q$-exponentially fast with mass $m>0$ if

$$
\limsup _{\|,\| \rightarrow \infty} \frac{\log \|\%, \psi\|}{\|x\|} \leqq-m
$$

Notice that if $\psi$ is an eigenfunction for an operator A as in (10) with (11), then $\psi$ decays exponentially fast with mass $m>0$ if it decay's q-exponentially fast with mass $m>0$ [Ag, Theorem 51$]$ Moreover, $\nabla \psi$ also decay's q-exponentially fast with mass $m>0$ (see Corollary 40)

We now adapt Theorem 21 in [DK] to our setting

Theorem 29. Let $A$ be a random operator as in (18) satisfying Assumption 1. Given $E_{0}>0, p>d$ and $m_{0}>0$, suppose

(P1) There exists $L_{0} \in 2 q \mathbb{N}$ such that

$$
\mathbb{P}\left\{\Lambda_{L_{0}}(0) \text { is }\left(m_{0}, E_{0}\right) \text {-regular }\right\} \geqq 1-\frac{1}{L_{0}^{p}}
$$

(P2) There exist $s>0, r>4 p+6 d$ and $C<\infty$ such that

$$
\mathbb{P}\left\{\operatorname{dist}\left(\sigma\left(A_{0, L}\right), E\right) \leqq \frac{1}{L^{\prime}}\right\} \leqq \frac{C|E|^{\frac{d}{2}-1}}{L^{\prime}}
$$

for all $E>0$ and $L \in 2 q \mathbb{N}$ with $\frac{1}{L^{\prime}}<E$

Then, given $m, 0<m<m_{0}$, there exists

$$
\mathscr{B}=\mathscr{B}\left(d, q, \varrho_{ \pm}, r_{u}, E_{0}, p, s, r, C, m_{0}, m\right)<\infty,
$$


depending only on the indicated constants, nondecreasing in $E_{0}$, such that, if we have $L_{0}>$ B we can find $\delta=\delta\left(E_{0}, L_{0}, m_{0}, m, s, C\right)>0$, so, with probability one, $A$ has only pure point spectum in $\left(E_{0}-\delta, E_{0}+\delta\right)$, and the corresponding eigenfunctions decay q-exponentially fast with mass $m>0$

Remark 30 A random operator $A_{y}$ as in (18) satisfying Assumption 1 always satisfies hypothesis (P2) for any $s>4 p+8 d$ and $r=s-2 d$, with

$$
C=Q \frac{U_{-}+2 U_{+}}{g U_{+}\left(1-g U_{+}\right) U_{-}}\|\rho\|_{\infty}, \quad Q=Q\left(d, \varrho_{0 . \pm}, r_{u}\right)<\infty
$$

This is just a special case of Theorem 23 Theorem 29 as stated is true with weaker hypotheses on $A_{y}$, the proof requires only (P2), the SLI, the EDI, and Theorem 43

Remark 31 Hypothesis (P1) says that we have localization in a large, but finite, volume, with high enough probability It is the starting hypothesis for the multiscale analysis The proof of Theorem 7 from Theorem 29 will consist of verifying (P1), using Assumption 2

Proof. Theorem 29 is proven as Theorem 21 in [DK], using the SLI and the EDI, with the following modifications.

1 We always take boxes $\Lambda_{L}(x)$ with $x \in q \mathbb{Z}^{d}$ and $L \in 2 q \mathbb{N}$

2 For two cubes $\Lambda_{L_{1}}\left(x_{1}\right)$ and $\Lambda_{L_{2}}\left(x_{2}\right)$ to be non-overlapping we require that $\left\|x_{1}-x_{2}\right\|>\frac{1}{2}\left(L_{1}+L_{2}+r_{u}\right)$ In this case the random operators $A_{r_{1}, L_{1}}$ and $A_{r_{2}, L_{2}}$ are independent

3 The probabilistic statement in [DK, Theorems 22 and 23 ] (called $R(L, m)$ in [DK, p 290]),

$$
\mathbb{P}\left\{\text { for any } E \in I \text { either } \Lambda_{L}(x) \text { or } \Lambda_{L}(y) \text { is }(m, E) \text {-regular }\right\} \geqq 1-\frac{1}{L^{2 p}},
$$

is now stated for any $x, y \in q \mathbb{Z}^{d}$ with $\|x-y\|>L+\frac{\iota_{u}}{2}$

4 The length scales $L_{h}^{\alpha}$ of [DK, Theorems 22 and 2.3] are now defined by $L_{k+1}=\left[L_{k}^{\gamma}\right]_{2 q}, k=0,1, \quad$, where $[t]_{2 q}=\sup \{n \in 2 q \mathbb{N}, n \leqq t\} \quad$ We also take $L=$ $\left[f^{y}\right]_{2 q}$ in [DK, Lemmas 41 and 42$]$

5 The basic tool for the proof of [DK, Lemma 4.2] in our setting is the SLI (164), it replaces inequality [DK, (4 1)].

6 We prove $q$-exponential decay of eigenfunctions in the analogues of [DK, Theorem 23 and Lemma 3 1] The basic tool in the proof of [DK, Lemma 3 1] is now the EDI (176). The basic facts about generalized eigenfunctions are given in Theorem 43, we use (244) with $\ell=q$

7 The fact that $\mathscr{B}$ can be chosen nondecreasing in $E_{0}$ follows from the fact that $E_{0}$ only appears in the proof when we use either (190), (164) or (176)

Theorem 29 suffices to prove Theorem 7, but Theorem 6 requires a somewhat different starting hypothesis, in which $m_{0}$ and $L_{0}$ are related

Theorem 32. Let $A$ be a random operator as in (18) satisfying Assumption 1 Given $E_{0}>0$ and $p>d$, suppose we have (P2) as in Theorem 29 and 
(Q1) There exists $L_{0} \in 2 q \mathbb{N}$ such that

$$
\mathbb{P}\left\{\Lambda_{L_{0}}(0) \text { is }\left(\frac{1 \log L_{0}}{L_{0}}, E_{0}\right) \text {-regular }\right\} \geqq 1-\frac{1}{L_{0}^{p}}
$$

for some $i>s+d-1$

Then, given $\xi, 0<\xi<1$, there exists

$$
\mathscr{C}=\mathscr{C}\left(d, q, \varrho_{ \pm}, r_{u}, E_{0}, p, s, r, C, \lambda, \xi\right)<\infty,
$$

depending only on the indicated constants, nondecreasing in $E_{0}$, such that, if we have $L_{0}>\mathscr{C}$, we can find $\delta=\delta\left(E_{0}, L_{0}, s, C, \lambda, \xi\right)>0$, so, with probability one, $A$ has only pure point spectrum in $\left(E_{0}-\delta, E_{0}+\delta\right)$, and the corresponding eigenfunctions decay q-exponentially fast with mass $\frac{\log L_{0}}{L_{0}}$

Proof This theorem is just Theorem 29 with $m_{0}=\frac{i \log L_{0}}{L_{0}}$, the same proof applies We need $i>s+d-1$ in $(\mathrm{Q} 1)$ to control the contribution of a singular region by the decay of a regular cube, using the SLI (164) and (P2) (see [DK, Lemma 4 2])

Remark 33 Notice that (193) is the same as

$$
\mathbb{P}\left\{\left\|\Gamma_{0, L_{0}} R_{0, L_{0}}\left(E_{0}\right) \%_{0}\right\|_{0, L_{0}} \leqq \frac{1}{L_{0}^{\frac{1}{2}}}\right\} \geqq 1-\frac{1}{L_{0}^{p}}
$$

Theorem 32 suffices to prove Theorem 6 with the stronger hypothesis $\eta>2 d$ in (23) To deal with the weaker hypothesis $\eta>d$ we adapt an argument of Spencer [Sp] to obtain the starting hypothesis (Q1) from a weaker (and easier to verify) hypothesis

Definition 34. Let the operator $A$ be as in (10) with (11) Given $v>0, E>0$, $x \in q \mathbb{Z}^{d}$ and $L \in 2 q \mathbb{N}, L \geqq 4 q$, we say that the cube $\Lambda_{L}(x)$ is $(1, E)$-suitable, if $E \notin \sigma\left(A_{1, L}\right)$ and

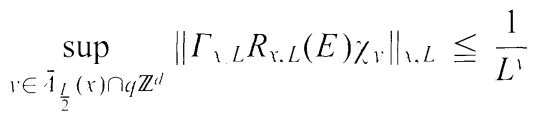

Theorem 35. Let $A$ be a random operator as in (18) satisfying Assumption 1 Given $E_{0}>0$ and $v>2(d-1)$, suppose

$$
\limsup _{L \rightarrow \infty} \mathbb{P}\left\{\Lambda_{L}(0) \text { is }\left(v, E_{0}\right) \text {-suitable }\right\}=1
$$

(H2) There exist $s \in(0, v-2(d-1)), r>10 d$ and $C<\propto$ such that

$$
\mathbb{P}\left\{\operatorname{dist}\left(\sigma\left(A_{0, L}\right), E_{0}\right) \leqq \frac{1}{L^{\zeta}}\right\} \leqq \frac{C\left|E_{0}\right|^{\frac{d}{2}-1}}{L^{\prime}}
$$

for all $L \in 2 q \mathbb{N}$ with $\frac{1}{L^{5}}<E_{0}$

Then there exists $\delta=\delta\left(d, q, Q_{+}, r_{u l}, E_{0}, 1, s, r, C\right)>0$, so, with probability one, A has only pure point spectrum in $\left(E_{0}-\delta, E_{0}+\delta\right)$, and the corresponding eigenfunctions decay q-exponentially fast 
Theorem 35 follows from Theorem 32 and from the following lemma

Lemma 36. Let $A$ be a random operator as in (18) satisfying Assumption 1 Given $E_{0}>0, r>d-1$ and $p>d$, suppose

(X1) There exists $L_{0} \in 2 q \mathbb{N}$ and $\delta_{0} \in(0,1)$ such that

$$
\mathbb{P}\left\{\Lambda_{L_{0}}(0) \text { is }\left(1, E_{0}\right) \text {-suitable }\right\} \geqq 1-\delta_{0}
$$

(X2) There exist $s \in(0, v-2(d-1)), r>p$ and $C<\infty$ such that

$$
\mathbb{P}\left\{\operatorname{dist}\left(\sigma\left(A_{0, L}, E_{0}\right)\right) \leqq \frac{1}{L^{\prime}}\right\} \leqq \frac{C\left|E_{0}\right|^{\frac{d}{2}-1}}{L^{\prime}}
$$

for all $L \in 2 q \mathbb{N}$ with $\frac{1}{L^{\prime}}<E_{0}$

Then there exist $\mathbb{Y}=\mathscr{Y}\left(d, q, \varrho_{ \pm}, r_{u}, E_{0}, v, s\right)<\infty$, depending only on the indicated constants, nondecreasing in $E_{0}$, such that given $\alpha>Y, \alpha \in \mathbb{N}$, we can find $\mathscr{Z}=\mathscr{Z}\left(d, q, \varrho_{ \pm}, r_{l l}, E_{0}, v, p, r, s, \alpha\right)<\infty$, depending only on the indicated constants, nondecreasing in $E_{0}$ and in $\alpha$, so if we have $L_{0}>\mathscr{Y}$ and $\delta_{0}<\left[2(2 \alpha)^{2 d}\right]^{-1}$ in (X1), and we set $L_{k+1}=x L_{k}, k=1,2$, we have

$$
\mathbb{P}\left\{\Lambda_{L_{h}}(0) \text { is }\left(1, E_{0}\right) \text {-suitable }\right\} \geqq 1-\frac{1}{L^{p}}
$$

for all $k \geqq \mathscr{K}$, where $\mathscr{K}=\mathscr{K}(\alpha, p)<\infty$

Proof The proof is by a multiscale analysis which combines ideas from [Sp, Theorem 1] and [DK] We set

$$
p_{L}=\mathbb{P}\left\{\Lambda_{L}(0) \text { is not }\left(v, E_{0}\right) \text {-suitable }\right\}
$$

The multiscale analysis is based on an induction argument, let $\ell \in 2 q \mathbb{N}, \alpha \in \mathbb{N}$ and $L=\alpha$. We set $\Xi_{L . /}=\Lambda_{L}(0) \cap \frac{1}{2} \mathbb{Z}^{d} \subset q \mathbb{Z}^{d}$, notice $\left|\Xi_{L, 1}\right| \leqq(2 \alpha)^{d}$ The induction step is similar to [DK, Lemma 4 1], it is based on the SLI, but only cubes in $\mathscr{C}_{L,\}}=\left\{\Lambda_{,}(x), x \in \Xi_{L, 1}\right\}$ are allowed, notice that $\Lambda_{L}(0) \subset \bigcup_{1 \in \Xi_{I},}, \bar{\Lambda}_{\frac{1}{2}}(x)$

Let $\tilde{F}_{L .}$ denote the event that either there are at least two non-overlapping cubes in $\mathscr{C}_{L . t}$ which are not $\left(v, E_{0}\right)$-suitable, or $\operatorname{dist}\left(\sigma\left(A_{x .3 /}, E_{0}\right)\right) \leqq \frac{1}{(3 /)^{\prime}}$ for some $x \in \Xi_{L, /}$, or $\operatorname{dist}\left(\sigma\left(A_{0, L}, E_{0}\right)\right) \leqq \frac{1}{L^{\prime}}$ As in [DK, Lemma 42$]$, we will show that

$$
\left\{\Lambda_{L}(0) \text { is not }\left(v, E_{0}\right) \text {-suitable }\right\} \subset \mathscr{F}_{L .},
$$

so

$$
\begin{aligned}
p_{L} \leqq \mathbb{P}\left(\mathscr{F}_{L . f}\right) & \leqq(2 \alpha)^{2 d} p_{\prime}^{2}+(2 \alpha)^{d} \frac{C\left|E_{0}\right|^{\frac{d}{2}-1}}{(3 \ell)^{\prime}}+\frac{C\left|E_{0}\right|^{\frac{d}{2}-1}}{L^{\prime}} \\
& \leqq(2 \alpha)^{2 d} p_{/}^{2}+\frac{C\left|E_{0}\right|^{\frac{d}{2}-1}}{L^{\prime}}\left(1+\frac{2^{d} \alpha^{d+1}}{3^{d}}\right)
\end{aligned}
$$

where we used (X2) to obtain the last two terms in (203)

To prove (202), we take $\ell>2 r_{u}+2 q$, so if $\omega \notin \tilde{F}_{L, /}$ there exist $x_{1} \in \Xi_{L, t}$, so that $\Lambda,(x)$ is $\left(1, E_{0}\right)$-suitable for any $x \in \Xi_{L . /} \backslash \Lambda_{3 /}\left(x_{1}\right)$ We control the "bad region" 
$\Lambda_{3 /}\left(x_{1}\right)$ as in [DK, Lemma 42 ], using the SLI (164) twice, we must require

$$
\left[\gamma_{E_{0}}(3 \ell)^{d-1}(3 /)^{s}\right]\left[\gamma^{\prime} E_{0} \ell^{d-1}\right]<\ell^{\prime}
$$

which is true for $y>s+2(d-1)$ and $\ell$ large $\left(\gamma_{E_{0}}\right.$ is given in $\left.(165)\right)$ We then have

$$
\sup _{l \in \bar{\Lambda}_{\frac{L}{2}}(0) \cap q \mathbb{Z}^{d}}\left\|\Gamma_{0 . L} R_{0, L}(E) \psi_{\nu}\right\|_{0, L} \leqq\left[\frac{\hat{\gamma}^{\prime} E_{0} l^{d-1}}{\ell^{v}}\right]^{N} L^{s},
$$

where $N$ is the number of times we are allowed to use the SLI on $\left(1, E_{0}\right)$-suitable boxes (without using the result for the control of a "bad box"). We have

$$
N \geqq \frac{\frac{L}{2}-q-6 \ell}{\frac{3 l}{4}-q} \geqq \frac{2}{3}(\alpha-14),
$$

so for $\Lambda_{L}(0)$ to be $\left(v, E_{0}\right)$-suitable we need

$$
\left[\frac{\gamma_{E_{0}} \ell^{d-1}}{\rho^{v}}\right]^{\frac{2}{3}(y-14)} L^{s}<\frac{1}{L^{v}}
$$

which is true for $\alpha$ sufficiently large since $v>d-1$ Thus (202) is proven

Let $\mathscr{F}$ be such that

$$
\frac{C\left|E_{0}\right|^{\frac{d}{2}-1}}{\mathscr{Z}^{\prime}-p}\left(1+\frac{2^{d} \alpha^{d+1}}{3^{d}}\right) \leqq \frac{1}{2}
$$

If we pick $L_{0}>\mathscr{Y}$, and set $L_{k+1}=\alpha L_{k}, k=1,2, \quad, p_{k}=p_{L_{k}}$, and $\gamma=(2 \alpha)^{2 d}$, it follows from (204) that

$$
p_{k+1} \leqq \gamma p_{k}^{2}+\frac{1}{2 L_{k+1}^{p}} \text { for } k=1,2, \ldots
$$

Notice first that if $p_{h}<\frac{1}{L_{h}^{p}}$, then

$$
p_{k+1} \leqq \gamma \frac{1}{L_{k}^{2 p}}+\frac{1}{2 L_{k+1}^{p}} \leqq \frac{2 \gamma \alpha^{p}}{L_{0}^{p}} \frac{1}{2 L_{k+1}^{p}}+\frac{1}{2 L_{k+1}^{p}} \leqq \frac{1}{L_{k+1}^{p}}
$$

if we require $\mathscr{Z}$ also to satisfy $\frac{2 ; q^{\prime \prime}}{\mathscr{y}^{p}} \leqq 1$. Now, suppose $p_{h+1} \geqq \frac{1}{L_{h+1}^{p}}$ for $k=1,2, \quad, n$ It follows from (210) that $i p_{k}^{2} \geqq \frac{1}{2 L_{k+1}^{p}}$ for $k=0,1,2, \quad, n-1$, so $p_{h+1} \leqq i p_{k}^{2}$ for $k=1,2, ., n$, so we have

$$
\frac{1}{\alpha_{k+1}^{p} L_{0}} \leqq p_{n+1} \leqq \frac{1}{2 \gamma}\left(2 \gamma p_{0}\right)^{2^{n+1}}
$$

Thus, if $2 \nmid p_{0}<1$, there must be $\mathscr{K}$ so $p_{k}<\frac{1}{L_{h}^{p}}$ for all $k \leqq \mathscr{K}$ Thus (200) is proven 
63 Boundary condition From periodic to Dirichlet The starting hypothesis (P1) for the multiscale analysis is formulated for operators with Dirichlet boundary condition But under the hypotheses of Theorems 6 and 7 the natural starting hypothesis is the analogue of (P1) for periodic boundary condition. The following lemma will enable us to go from periodic boundary condition to Dirichlet boundary condition

Let $A_{q}$ be as in (18) satisfying Assumption 1 Given $x \in q \mathbb{Z}^{d}$ and $L \in 2 q \mathbb{N}$, we set (with the notation of (103))

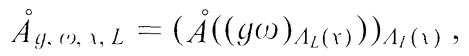

which is a random operator by Theorem 38 We write $\stackrel{\circ}{R}_{g,(1)}, r, L(z)$ for its resolvent

Lemma 37. Let $A_{g}$ be as in (18) satisfying Assumption 1 Let $E>0, x \in q \mathbb{Z}^{d}$ and $L \in 2 q \mathbb{N}, L \geqq 4 q$, set $\hat{L}=L+\left[2 r_{u}\right]_{2 q}+2 q$ If $\omega$ is such that $E \notin \sigma\left(A_{\mathcal{g}_{(}(1),, L} \cup\right.$ $\sigma\left(A_{\text {g. (1), }, . \hat{L}}^{\circ}\right)$, then

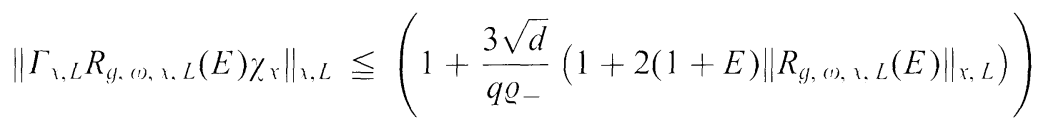

$$
\begin{aligned}
& \times\left\|\Gamma_{\gamma, L} R_{y,(1), r, \hat{L}}^{\circ}(E) \psi_{r}\right\|_{r, \hat{L}}
\end{aligned}
$$

Proof From Lemma 24 and the definition of periodic and Dirichlet boundary condition, plus the choice of $\hat{L}$ which ensures that

$$
\varrho_{(y(1))_{L_{L}(1)}}(y)=\varrho_{g,(1)}(y) \text { for all } y \in \Lambda_{L}(x),
$$

we get the following analogue to the SRI (154)

$$
\begin{aligned}
& \stackrel{\circ}{R}_{y,(1), r, \hat{L}}(E) J_{r, L}^{\lambda, \hat{L}} \Phi_{\mathrm{r}, L}=J_{r, L}^{r, \hat{L}} \Phi_{\uparrow, L} R_{\mathrm{g},(1), \mathrm{r}, L}(E)
\end{aligned}
$$

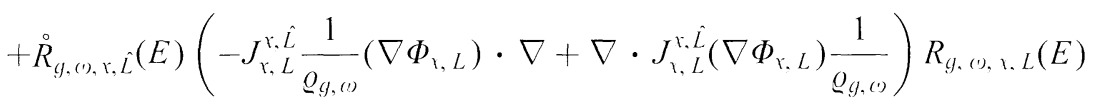

as quadratic forms on $L^{2}\left(\Lambda_{\lambda}, \hat{L}\right) \times L^{2}\left(\Lambda_{\lambda, L}\right)$

Proceeding as in the proof of Lemma 26, we get (we omit $g$ and $(1)$ from the notation)

$$
\begin{aligned}
& \left\|\psi_{r} \stackrel{\circ}{R}, \hat{L}_{r}(E) J_{\uparrow, L}^{r, \hat{L}} \frac{1}{\varrho}\left(\nabla \Phi_{r, L}\right) \cdot \nabla R_{r, L}(E) \Gamma_{r, L}\right\|_{r, L}^{r, \hat{L}} \\
& \leqq \frac{3 \sqrt{d}}{q Q-}\left\|\hat{\Gamma}_{x, L} \nabla R_{\imath, L}(E) \Gamma_{r, L}\right\|_{r, L}\left\|\chi_{\imath} \stackrel{\circ}{R}, L_{L}(E) \Gamma_{r, L}\right\|_{r, \hat{L}} \\
& \leqq \frac{3 \sqrt{d}}{q \varrho-} \Theta_{\frac{q}{4}}\left(1+(1+E)\left\|R_{r . L}(E)\right\|_{r, L}\right)\left\|\Gamma_{r, L} \stackrel{\circ}{R}_{g \cdot(\omega, x, \hat{L}}(E) \psi_{r}\right\|_{r, \hat{L}}
\end{aligned}
$$


and

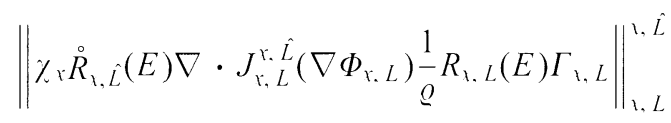

$$
\begin{aligned}
& \leqq \frac{3 \sqrt{d}}{q Q_{-}}\left\|\hat{\Gamma}_{r, L} R_{\curlyvee, L}(E) \Gamma_{r, L}\right\|_{\mathrm{r}, L}\left\|\hat{\Gamma}_{\mathrm{r}, L} \nabla \stackrel{\circ}{R}_{\mathrm{r}, \hat{L}}(E) \psi_{r}\right\|_{\mathrm{r}, \hat{L}}
\end{aligned}
$$

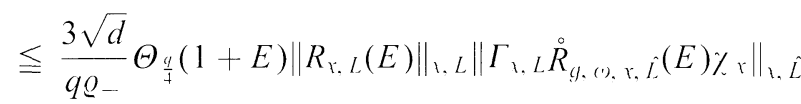

Equation (214) now follows from (216),(217) and (218)

64 Localization in a specified interval We now prove Theorem 7 from Theorem 29 Let the random operator $A_{g}$ and $a_{1}, a_{2}, g_{1}$ be as in Theorem 7, let us fix an upper bound $\tilde{\rho}$ for $\|\rho\|_{\infty}$, and set

$$
p_{1}^{\prime}=\mu\left(\left(\frac{g_{1}}{g}, 1\right]\right)
$$

(P2) follows from Theorem 23 (see Remark 30) To prove Theorem 7, since $\mathscr{B}$ in Theorem 29 is nondecreasing in $E_{0}$, we need only to verify (P1) uniformly on $E \in\left[a_{2}, a(g)\right]$ for some sufficiently large $L_{0}$ and all sufficiently small $p_{1}^{\prime}$, this will be done using Assumption 2 It thus suffices to show that we can find $m_{0}>0$ and $p>d$ such that

$$
\limsup _{p_{1}^{\prime} \rightarrow 0} \inf _{E \in\left[a_{2}, a(g)\right]} \mathbb{P}\left\{\Lambda_{l}(0) \text { is }\left(m_{0}, E\right) \text {-regular }\right\}>1-\frac{1}{L^{p}}
$$

for some sufficiently large $L \in 2 q \mathbb{N}$

We will prove more Let us fix $p>d$ For a given $L \in 2 q \mathbb{N}, L \geqq 4 q$, we set $\hat{L}$ as in Lemma 37 and define the event

$$
\mathscr{E}_{L}=\left\{\omega_{l} \leqq \frac{g_{1}}{g} \quad \text { for all } i \in \mathbb{Z}^{d} \cap \check{\Lambda}_{L}(0)\right\}
$$

Since $a\left(g_{1}\right)=a_{1}$, we can conclude from Theorem 3, Lemma 19 and Theorem 14 that

$$
\left(a_{1}, b(g)\right) \subset \mathbb{R} \backslash \sigma\left(\dot{A}_{g,(1,0 . \hat{L} .}\right) \text { for all } \omega \in \mathscr{E}_{L}
$$

It now follows from Lemma 18 (with $r=q$ ), (158) and (159), that for (1) $\in \mathscr{E}_{L}$ and $E \in\left(a_{1}, a(g)\right]$ we have

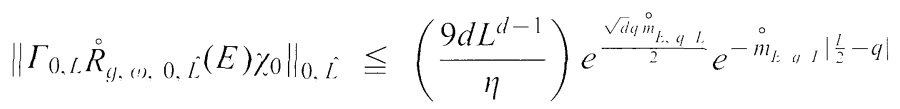

$$
\begin{aligned}
& \leqq\left(\frac{9 d L^{d-1}}{\eta}\right) e^{\left(\frac{\sqrt{d}}{2}+1\right) \dot{\circ} m_{i, 4 l}} e^{-\dot{m}_{t, 4} \frac{l}{2}}
\end{aligned}
$$

where $\dot{m}_{E, q, \hat{L}}$ is given in (93) and $\eta=E-a_{1}$ since $a(g)-a_{1} \leqq b(g)-a(g)$, we assume $\hat{L}>2(2 q+8)$ 
If we now take $E \in\left[a_{2}, a(g)\right]$, so $a_{2}-a_{1} \leqq \eta \leqq a(g)-a \leqq a \delta_{+}(g) g$ (we used (21)), we have

$$
M \equiv \frac{a_{2}-a_{1}}{4(4 \sqrt{d}+1)\left[\varrho_{-}^{-1}+a+a \delta_{+}(g) g\right]} \leqq \dot{m}_{E . q . \hat{L}} \leqq \frac{1}{4},
$$

so

$$
\left\|\Gamma_{0, L} \stackrel{\circ}{R}_{\{,(1), 0 . \hat{L}}(E) \%_{0}\right\|_{0, \hat{L}} \leqq\left(\frac{9 d L^{d-1}}{a_{2}-a_{1}}\right) e^{\frac{(\sqrt{d}-2) k}{8}} e^{-M \frac{L}{2}}
$$

At this point we introduce the events

$$
\|_{E, L}=\left\{\operatorname{dist}\left(\sigma\left(A_{0, L}, E\right)\right)>\frac{1}{L^{2 p+2 d}}\right\},
$$

and set $\mathscr{G}_{E, L}=\mathscr{E}_{L} \cap \mathscr{H}_{E, I}$. If $E \in\left[a_{2}, a(g)\right]$ and $\left(\omega \in \mathscr{G}_{E . L}\right.$, we can apply Lemma $37,(21),(225)$ and (226) to obtain

$$
\begin{aligned}
& \left\|\Gamma_{0, L} R_{\mathscr{G}(1)} 0, L(E) \%_{0}\right\|_{0, L} \\
& \quad \leqq\left(\frac{9 d L^{d-1}}{a_{2}-a_{1}}\right) e^{\frac{(\sqrt{d} 2 k d}{8}}\left(1+\frac{3 \sqrt{d}}{q Q_{-}}\left(1+2\left(1+\frac{a}{1-g U_{+}}\right) L^{2 p+2 d}\right)\right) e^{-M \frac{L}{2}}
\end{aligned}
$$

Thus, if we take $m_{0}=\frac{M}{2}$, we can find

$$
T_{1}=T_{1}\left(d, q, g, \varrho_{ \pm}, r_{u}, a, a_{1}, a_{2}, p\right)<\infty,
$$

such that if $(1) \in \mathscr{G}_{E . L}$, with $E \in\left[a_{2}, a(g)\right]$, we have that the cube $\Lambda_{L}(0)$ is $\left(m_{0}, E\right)$ regular for any $L>T_{1}$ To conclude the proof of Theorem 7, notice that from (221), (219), (226) and (191) we get, for $\frac{1}{L^{2 p+2 d}}<E \leqq a(g)$,

$$
\begin{aligned}
\mathbb{P}\left(\mathscr{G}_{E, L}\right) & \geqq 1-p_{1}^{\prime} \hat{L}^{d}-\frac{C}{L^{2 p}}\left(\frac{a}{1-g U_{+}}\right)^{\frac{d}{2}-1} \\
& \geqq 1-p_{1}^{\prime} \hat{L}^{d}-\frac{1}{2 L^{p}}
\end{aligned}
$$

with (229) valid for $L>T_{2}$, where

$$
T_{2}=T_{2}\left(d, q, g, \varrho_{0} \pm, U_{ \pm}, r_{u}, a, \tilde{\rho}, p\right)<\infty
$$

We can conclude that

$$
\inf _{E \in\left[a_{1}, a(g)\right]} \mathbb{P}\left\{\Lambda_{L}(0) \text { is }\left(m_{0}, E\right) \text {-regular }\right\} \geqq \inf _{E \in\left[a_{1}, a(g)\right]} \mathbb{P}\left(\mathscr{G}_{E, L}\right)>1-\frac{1}{L^{p}}
$$

for all $L>\max \left\{T_{1}, T_{2}\right\}$ and $p_{1}^{\prime}<\frac{1}{2 L^{p+d}}$, so (220) follows

Theorem 7 is proven 
65 Localization at the edge We now prove Theorem 6 from either Theorem 32 or Theorem 35 Let the random operator $A_{y}$ be as in Theorem 6 Since we have $(\mathrm{P} 2)$ and $(\mathrm{H} 2)$ in the form given in Remark 30, to prove Theorem 6 it suffices to establish either (Q1) or $(\mathrm{H} 1)$ for $E_{0}=a(g)$

So let us fix $p>d, i>4 p+9 d-1$, and let $a_{1}, g_{1}, p_{1}^{\prime}, \hat{L}, \mathscr{E}_{L}, \mathscr{H}_{E . L}, \mathscr{G}_{E, L}$ be as in Subsect 64 Notice that (23) can be written as

$$
p_{1}^{\prime} \leqq K\left(\frac{g-g_{1}}{g}\right)^{\eta}
$$

For $\left(\omega \in \mathscr{G}_{a(g), L}\right.$, we have (227) with $a_{2}=a(g)$ and $M$ as in (224) If we choose $a_{1}=a(g)-\kappa \cdot \frac{\log L}{L}$, with $\kappa \geqq 1$ and $L$ such that $a<a_{1}$ and $a(g)-a_{1} \leqq b(g)-$ $a(g)$, we get

$$
\left\|\Gamma_{0, L} R_{g,(\omega, 0, L}(a(g)) \%_{0}\right\|_{0, L} \leqq C_{1} L^{-\frac{c h}{2}+2 p+3 d} \leqq L^{-\frac{1}{2}},
$$

where the constants $C_{1}$ and $c$ can be read from (227) and (224), the last inequality in (233) is valid for $k>c^{-1}(\lambda+4 p+6 d)$ and $L$ sufficiently large

If $L>T_{2}$ (with $T_{2}$ given in (230)), it follows from (229) and (232) that

$$
\begin{aligned}
\mathbb{P}\left(\mathscr{G}_{a(g) . L}\right) & \geqq 1-K\left(\frac{g-g_{1}}{g}\right)^{\eta} \hat{L}^{d}-\frac{1}{2 L^{p}} \\
& \geqq 1-K\left(\frac{\kappa}{g \delta_{-}(g) a}\right)^{\eta}\left(\frac{\log L}{L}\right)^{\eta} \hat{L}^{d}-\frac{1}{2 L^{p}},
\end{aligned}
$$

where we used (135) to get (235)

If $\eta>2 d$, it follows immediately from (233) and (235) that the starting hypothesis (Q1) holds for all $L$ sufficiently large, so Theorem 6 follows from Theorem 32

If we only have $\eta>d$ we prove (H1) Let $r>10 d, s=r+2 d$ and $v>s+$ $2(d-1)$. For $(1) \in \mathscr{G}_{a(g) . L}$ (we take $p=r$ in $\left.(226)\right)$, we have

$$
\sup _{v \in \overline{1}_{\frac{l}{2}}(0) \cap q \mathbb{Z}^{d}}\left\|\Gamma_{0, L} R_{y,(1) 0, L}(a(g)) \psi_{v}\right\|_{0, L} \leqq C_{1} L^{-\frac{c h}{4}+2 p+3 d} \leqq L^{-v},
$$

where (236) is derived in the same way as (233), with the same constants $C_{1}$ and $c$, with the last inequality valid for $k>4 c^{-1}(v+2 p+3 d)$ and $L$ sufficiently large Since we have (235) and $\eta>d$, the starting hypothesis (H1) follows, so Theorem 6 follows from Theorem 35

Theorem 6 is proven

\section{A. Measurability of Operator Valued Functions}

In this appendix we will prove the desired measurability properties for our random operators A similar result, with somewhat different technical assumptions appropriate to random Schrödinger operators, was proved by Kirsch and Martinelli [KM1, Proposition 6]

We say that a mapping ( $) \mapsto A_{(\prime)}$ from a probability space $\Omega$ to nonnegative self-adjoint operators on a separable Hilbert space $\mathscr{H}$ is measurable if the mappings (1) $\in \Omega \mapsto f\left(A_{(\prime)}\right) \in \mathscr{B}(\mathscr{H})$ are weakly (and hence strongly) measurable for all bounded measurable functions $f$ on $\mathbb{R}$ In this case $A_{\left({ }^{\prime}\right)}$ is called a random operator 
Theorem 38. Let (1) $\mapsto A_{(1)}$ be a mapping from a probability space $\Omega$ to nonnegative self-adjoint operators on a separable Hilbert space $\mathscr{H}$, such that

$$
S \leqq A_{(\prime)} \leqq c S \text { with probability one },
$$

for some nonnegative self-adjoint operator $S$ and constant $c<\infty$ Let $S_{\text {(") }}$ and $\mathscr{P}$ be the corresponding quadratic forms, $\mathscr{Z}=\mathscr{L}(\mathscr{S})$ being the common domain Suppose $(1) \mapsto \mathscr{A}_{(1)}(\psi)$ is measurable for all $\psi \in \mathscr{L}$, then $A_{(\prime)}$ is a random operator

Proof Let $R_{\left({ }^{\prime}\right)}=\left(A_{(\prime)}+I\right)^{-1}$, it suffices to prove that the mapping $(1) \mapsto R_{(\prime)}$ is weakly measurable (e g, the argument in [PF, p 40]) We proceed as in [KM1, Proof of Proposition 6] Using (237), we have that (with probability one)

$$
\left(A_{(\prime)}+I\right)^{-1}=(S+I)^{-\frac{1}{2}}\left((S+I)^{-\frac{1}{2}}\left(A_{\left(^{\prime}\right)}-S\right)(S+I)^{-\frac{1}{2}}+I\right)^{-1}(S+I)^{-\frac{1}{2}},
$$

where $(S+I)^{-\frac{1}{2}}\left(A_{(\prime)}-S\right)(S+I)^{-\frac{1}{2}}$ is a bounded nonnegative operator and the map ( $) \in \Omega \mapsto(S+I)^{-\frac{1}{2}}\left(A_{(\prime)}-S\right)(S+I)^{-\frac{1}{2}} \in \mathscr{B}(\mathscr{H})$ is clearly weakly measurable, hence measurable by [KM1, Lemma after Proposition 6]

\section{B. Some Results on Elliptic Operators}

Divergence operators of the form (18) can have nonsmooth coefficient $Q(x)$ that implies, in particular, that the standard functional space $C_{0}^{\infty}$ cannot be used even as a subset of the domains of such operators. In this appendix we provide some tools to treat these operators

\section{B I An interior estimate}

Proposition 39 (|GT, Problem 8.2]). Let $A$ be an operator of the form (10) satisfying the bounds (11) For any $\delta>0$ there exists a constant $\Theta_{\delta}=\Theta\left(d, \varrho_{ \pm}, \delta\right)$ $<\propto$, depending only, on the indicated parameters, such that for any open subset $\Omega$ of $\mathbb{R}^{d}$, if $u \in W^{1,2}(\Omega)$ is a weak solution for the equation $A u=f$ in $\Omega$, with $f \in L^{2}(\Omega)$, we have

$$
\|\nabla u\|_{2 . \Omega^{\prime}} \leqq \Theta_{\partial}\left[\|u\|_{2, \Omega}+\|f\|_{2 . \Omega}\right]
$$

for any $\Omega^{\prime} \subset \Omega$ with $\operatorname{dist}\left(\Omega^{\prime}, \hat{c} \Omega\right) \geqq \delta$

We have the following immediate corollary

Corollary 40. Let $A$ be an operator of the form (10) satisfying the bounds (11), and let $\varphi$ be an eigenfunction for $A$ Suppose $\varphi$ has exponentially decaying local $L^{2}$-norms, $i$ e, $\left\|\%_{1}, \varphi\right\|_{2}$ decals exponentially as $\|x\| \rightarrow \infty$ for some $\ell>0$ Then $\nabla \varphi$ also has exponentially. decaying local $L^{2}$-norms

Proposition 39 has the following obvious extension to $\mathbb{R}^{d}$

Proposition 41. Let $A$ be an operator of the form (10) satisfing the bounds (11) If $u \in W^{1.2}\left(\mathbb{R}^{d}\right)$ is a weak solution for the equation $A u=f$ in $\mathbb{R}^{d}$, with $f \in$ $L^{2}\left(\mathbb{R}^{d}\right)$, we have

$$
\|\nabla u\|_{2} \leqq \Theta_{\infty}\left[\|u\|_{2}+\|f\|_{2}\right]
$$

with $\Theta_{\infty}=\inf _{\partial>0} \Theta\left(d, \varrho_{ \pm}, \delta\right)$ 
$B 2$ Generalized eigenfunctions Let $A$ be an operator of the form (10) satisfying the bounds (11) Given $z \in \mathbb{C}$, a measurable function $\varphi$ on $\mathbb{R}^{d}$ will be called a generalized eigenfunction for $z$ if $\gamma_{\Omega} \varphi \in W^{1.2}(\Omega)$ for all bounded domains $\Omega$ in $\mathbb{R}^{d}$ and $\varphi$ is a weak solution for the equation $A \psi=z \psi$ on $\mathbb{R}^{d}$, i.e ,

$$
\left\langle\nabla \psi, \frac{1}{\varrho} \nabla \varphi\right\rangle=z\langle\psi, \varphi\rangle \text { for all } \psi \in C_{0}^{1}\left(\mathbb{R}^{d}\right)
$$

To obtain properties of generalized eigenfunctions we use the following estimates on the Green's functions of divergence operators with nonsmooth uniformly bounded coefficients [D, Corollary 328 and Lemma 342 2]

Proposition 42. Let $A$ be an operator of the form (10) satisfying the bounds (11), let $\ell>d / 2$ and $\mu>0$ Then the operator $R^{\prime}=(\mu I+A)^{--1}$ has a kernel $R^{\prime}(x, y)$ satisfying the following a priori estimate

$$
0 \leqq R^{\prime}(x, y) \leqq c e^{-\beta|1-\psi|} \quad \text { for all } x, y \in \mathbb{R}^{d} \text {, }
$$

whene the constant $c$ depends only on $d, Q_{ \pm}, \ell$ and $\mu$, and $\beta=\sqrt{\frac{\mu Q_{-}}{2 d}}$

Theorem 43. Let $A$ be an operator of the form (10) satisfying the bounds (11), $\rho(d i)$ its the spectral measure Let $w(x)=\left(|x|^{d^{\prime}}+1\right)^{-1}, d^{\prime}>d$ Then for $\rho(d i)$ almost all i. A has a generalized eigenfunction $\varphi$; satisfying

$$
\int_{\mathbb{R}^{d}}|\varphi,(x)|^{2} w(x) d x<\infty
$$

so for any $t \in \mathbb{N}$ we have

$$
\|\%, \varphi \varphi,\| \leqq C_{,}\left(|x|^{d^{\prime}}+1\right) \quad \text { for all } x \in \ell \mathbb{Z}^{d}
$$

for some constant $C_{1}<\infty$ depending only on $t, d, Q_{ \pm}$and the LHS of (243) Moreover, for such $\varphi$, we also have

$$
|\varphi ;(x)|^{2} \leqq C\left(|x|^{d^{\prime}}+1\right) \quad \text { for all } x \in \mathbb{R}^{d},
$$

for some constant $C<\infty$ depending only' on $d, \varrho_{ \pm}$and the LHS of (243)

Proof Notice that in view of Proposition $42 R^{\prime}=(A+\mu I)^{-\ell}$ for $\ell>d / 2$ is a Carleman operator such that

$$
\int_{\mathbb{R}^{\prime}} \int_{\mathbb{R}^{\prime}}\left|R^{\prime}(x, v)\right|^{2} w(x) d x d y<\infty
$$

This and [B, Theorem V 4 1] imply (243), from which (244) follows The estimate (245) follows from (244) and [Ag, Theorem 5 1] 


\section{Lemmas on Convergence of Operators}

Lemma 44. Let $S_{n}^{+}, S_{n}^{-}, K_{n}, n \geqq 1$ be sequences of self-adjoint bounded operators in a Hilbert space $\mathscr{H}$ such that for some constant $C<\infty$ we have

$$
0 \leqq S_{n}^{ \pm} \leqq C I \quad \text { for all } n \geqq 1
$$

and

$$
\lim _{n \rightarrow \infty}\left\langle\varphi, S_{n}^{ \pm} \varphi\right\rangle=0 \quad \text { for all } \varphi \in \mathscr{H}
$$

Then

$$
\lim _{n \rightarrow \infty} S_{n}^{ \pm} \varphi=0 \quad \text { for all } \varphi \in \mathscr{H}
$$

If in addition we have

$$
-S_{n}^{-} \leqq K_{n} \leqq S_{n}^{+}
$$

then

$$
\lim _{n \rightarrow \infty} K_{n} \varphi=0 \quad \text { for all } \varphi \in \mathscr{H}
$$

Proof Notice that if $S_{n}$ stands for either $S_{n}^{-}$or $S_{n}^{+}$then it follows from (247) that

$$
S_{n}^{2}=\sqrt{S_{n}} S_{n} \sqrt{S_{n}} \leqq \sqrt{S_{n}} C \sqrt{S_{n}}=C S_{n} .
$$

This together with (248) implies that $\left\|S_{n} \varphi\right\|^{2} \leqq C\left\langle\varphi, S_{n} \varphi\right\rangle \rightarrow 0$ as $n \rightarrow \infty$, so (249) is true.

Observe now that in view of (247) and (248) the operators $G_{n}=K_{n}+S_{n}^{-}$satisfy

$$
0 \leqq G_{n} \leqq S_{n}^{--}+S_{n}^{+} \leqq 2 C I
$$

and

$$
\lim _{n \rightarrow \infty}\left\langle\varphi, G_{n} \varphi\right\rangle=0, \quad \varphi \in \mathscr{H}
$$

Hence, using (249), we obtain

$$
\lim _{n \rightarrow \infty} G_{n} \varphi=0, \quad \varphi \in \mathscr{H} .
$$

Recalling now that $K_{n}=G_{n}-S_{n}$ and using (249) and (252) we obtain (251).

Given a nonnegative operator $B$ the associated quadratic form will be denoted by

$$
B[\varphi, \psi] \equiv\langle\sqrt{B} \varphi, \sqrt{B} \psi\rangle, \quad \varphi, \psi \in \mathscr{L}(\sqrt{B})
$$

We will write $B[\varphi] \equiv B[\varphi, \varphi]$

Lemma 45. Let $S$ and $S_{n}, S_{n}^{ \pm}(n \geqq 1)$ be self-adjoint operators in a Hilbert space $\mathscr{H}$ such that

$$
S-s_{n}^{-} \leqq S_{n} \leqq S+s_{n}^{+}
$$

(the sums of possibly unbounded operators being understood in the quadratic form sense),

$$
0 \leqq s_{n}^{ \pm} \leqq \alpha^{ \pm} S \quad \text { for some } 0 \leqq \alpha^{-}<1, \quad 0 \leqq \alpha^{+}<\infty,
$$

and

$$
\lim _{n \rightarrow \infty} s_{n}^{ \pm}[\varphi]=0 \quad \text { for all } \varphi \in \mathscr{L},
$$


where $\mathscr{I} \subset \mathscr{L}(\sqrt{S})$ is a core for the operator $\sqrt{S}$ Then

$$
\lim _{n \rightarrow \infty}\left(I+S_{n}\right)^{-1} \psi=(I+S)^{-1} \psi \text { for all } \psi \in \mathscr{H},
$$

so $S_{n}$ converge to $S$ in the strong resolvent sense and we have

$$
\overline{\bigcup_{n \geqq 1} \sigma\left(S_{n}\right)} \supset \sigma(S)
$$

Proof We set $R_{n}=\left(I+S_{n}\right)^{-1}, R=(I+S)^{-1}$, and notice that $-r_{n}^{-} \leqq R_{n}-R \leqq$ $r_{n}^{+}$, where

$$
\begin{array}{ll}
r_{n}^{+}=\rho_{n}^{+}-(I+S)^{-1} \geqq 0, & \rho_{n}^{+}=\left(I+S-s_{n}^{-}\right)^{-1}, \\
r_{n}^{-}=(I+S)^{-1}-\rho_{n}^{-} \geqq 0, & \rho_{n}^{-}=\left(I+S+s_{n}^{+}\right)^{-1}
\end{array}
$$

Clearly

$$
\left\|R_{n}\right\|,\|R\|,\left\|r_{n}^{ \pm}\right\|,\left\|\rho_{n}^{ \pm}\right\| \leqq 1,
$$

and, in view of (253) and (254),

$$
\begin{aligned}
& \mathscr{L}\left(\sqrt{S_{n}}\right)=\mathscr{L}\left(\sqrt{S \pm s_{n}^{ \pm}}\right)=\mathscr{Z}(\sqrt{S}), \\
& R \varphi, \rho_{n}^{ \pm} \varphi \in \mathscr{L}(\sqrt{S}), \quad \varphi \in \mathscr{H}
\end{aligned}
$$

In addition, (255) together with $s_{n}^{ \pm} \leqq \alpha^{ \pm} S$ imply

$$
\lim _{n \rightarrow \infty} s_{n}^{ \pm}[\varphi]=0 \quad \text { for all } \varphi \in \mathscr{Y}(\sqrt{S})
$$

In view of Lemma 44, to prove (256) it is sufficient to verify that

$$
\lim _{n \rightarrow \infty}\left\langle r_{n}^{ \pm} \psi, \psi\right\rangle=0 \quad \text { for all } \psi \in \mathscr{H}
$$

Let us consider $r_{n}^{+}$Observe that from (258),(259) and (260) we have

$$
\begin{aligned}
\left\langle r_{n}^{+} \psi, \psi\right\rangle & =\left\langle\rho_{n}^{+} \psi,(I+S) R \psi\right\rangle-\left\langle\left(I+S-s_{n}^{-}\right) \rho_{n}^{+} \psi, R \psi\right\rangle \\
& =(I+S)\left[\rho_{n}^{+} \psi, R \psi\right]-\left(I+S-s_{n}^{-}\right)\left[\rho_{n}^{+} \psi, R \psi\right] \\
& =s_{n}^{-}\left[\rho_{n}^{+} \psi, R \psi\right]=\left\langle\sqrt{s_{n}^{-}} \rho_{n}^{+} \psi, \sqrt{s_{n}^{-}} R \psi\right\rangle, \quad \psi \in \mathscr{H}
\end{aligned}
$$

The norms of the vectors $\sqrt{s_{n}^{-}} \rho_{n}^{+} \psi$ and $\sqrt{s_{n}^{-}} R \psi$ can be estimated as follows. Since $s_{n}^{-} \leqq \alpha^{-} S$, we have

$$
s_{n}^{-} \leqq \frac{\alpha^{-}}{1-\alpha^{-}}\left(S-s_{n}^{-}\right)
$$

Notice that for any nonnegative operator $K$ we have

$$
K \leqq(I+K)^{2}
$$

Combining (264) with the last inequality for $K=S-s_{n}^{-}$, we obtain

$$
s_{n}^{-} \leqq \frac{\alpha^{-}}{1-\alpha^{-}}\left(I+S-s_{n}^{-}\right)^{2}=\frac{\alpha^{-}}{1-\alpha^{-}}\left(\rho_{n}^{+}\right)^{-2},
$$


which readily implies

$$
\rho_{n}^{+} s_{n}^{-} \rho_{n}^{+} \leqq \frac{\alpha^{-}}{1-\alpha^{-}} I \text { and }\left\|\sqrt{s_{n}^{-}} \rho_{n}^{+}\right\|^{2} \leqq \frac{\alpha^{-}}{1-\alpha^{-}}
$$

It now follows from (263) and (265) that for $\psi \in \mathscr{H}$ we have

$$
\left\langle r_{n}^{+} \psi, \psi\right\rangle^{2} \leqq\left\|\sqrt{s_{n}^{-}} R \psi\right\|^{2}\left\|\sqrt{s_{n}^{-}} \rho_{n}^{+}\right\|^{2}\|\psi\|^{2} \leqq \frac{\alpha^{-}}{1-\alpha^{-}} s_{n}^{-}[R \psi]\|\psi\|^{2}
$$

The last inequality together with (260) and (261) imply (262) for $r_{n}^{+}$The proof of this statement for $r_{n}^{-}$is analogous, using $s_{n}^{+} \leqq \frac{y^{+}}{1+x^{+}}\left(S+s_{n}^{+}\right)$.

To prove (257) it is sufficient to prove the similar inclusion for the resolvents $R_{n}$ and $R$, namely

$$
\overline{\bigcup_{n \geqq 1} \sigma\left(R_{n}\right)} \supset \sigma(R)
$$

Suppose that $i \in \sigma(R)$ Then for any positive $\gamma$ there exists a vector $\psi \in \mathscr{H}$ such that $\|R \psi-\hat{\lambda} \psi\| \leqq \gamma,\|\psi\|=1$. In view of the strong convergence of $R_{n}$ to $R$ this implies that for sufficiently large $n$ we have $\left\|R_{n} \psi-\lambda \psi\right\| \leqq 2 \gamma$ Since $i$ is an arbitrary number the last inequality implies (266) and, hence, the desired inclusion (257)

Acknowledgements The authors thank P Kuchment, S Molchanov, M Schechter and B Vainberg for useful discussions and suggestions

\section{References}

[Ag] Agmon, S : Lectures on Exponential Decay of Solutions of Second-Order Elliptic Equation Mathematical Notes 29, Princeton NJ: Princeton University Press, 1982

[An1] Anderson, PW: Absense of Diffusion in Certain Random Lattice Phys Rev 109, $1492-1505$ (1958)

[An2] Andesson, P W A Question of Classical Localization A Theory of White Paint Philosophical Magazine B53, 505-509 (1958)

[B] Berezanskii, Iu M: Expansions in Eigenfuncions of Selfadjoint Operators Providence, RI: AMS, 1968

[CH] Combes, $\mathrm{J}$ and Hislop, P: Localization for some Continuous, Random Hamiltonians in d-dimensions J Funct Anal 124, 149-180 (1994)

[D] Davies, E B : Heat Kernels and Spectral Theory Cambridge: Cambridge University Press, 1989

[DE] Development and Applications of Materials Exhibiting Photonic Band Gaps, J Optical Socicty of America B10, 280 413 (1993)

[DK] Dreifus, $\mathrm{H}$ and Klein, A : A new proof of localization in the Anderson tight binding model Commun Math Phys 124, 285-299 (1989)

[Ea] Eastham, M: The Spectral Theory of Periodic Differential Equations Scottish Academic Press, 1973

[FK1] Figotin, A and Klein, A : Localization Phenomenon in Gaps of the Spectrum of Random Lattice Operators J Stat Phys 75, 997-1021 (1994)

[FK2] Figotin, A and Klein, A : Localization of Electiomagnetic and Acoustic Waves in Random Media Lattice Model J Stat Phys 76, 985-1003 (1994)

[FK3] Figotin, A and Klein, A : Localization of Classical Waves II: Electromagnetic Waves Preprint

[FK4] Figotin, A and Klein, A Localized Classical Waves Created by Defects J Stat Phys, to appear 
[FKul] Figotin, A and Kuchment, P : Band-Gap Structure of Spectra of Periodic Dielectric and Acoustic Media I Scalar Model SIAM J Appl Math 56, 68-88 (1996)

[FKu2] Figotin, A and Kuchment, P : Band-Gap Structure of Spectra of Perrodic Dielectric and Acoustic Media II 2D Photonic Crystals SIAM J Appl Math, to appear

[GT] Gilbarg, D and Tiudinger, N S : Elliptic Partial Differential Equations of Second Order Berlin-Heidelberg-New York: Springer-Verlag, 1983

[HM] Holden, $\mathrm{H}$ and Martinelli, F : On Absence of Diffusion near the Bottom of the Spectrum for a Random Schrödinger Operator on $L^{2}\left(\mathbb{R}^{\prime \prime}\right)$ Commun Math Phys 93, 197-217 (1984)

[J1] John, S : Localization of Light Phys Today, May 1991

[J2] John, S: The Localization of Light In: "Photonic Band Gaps and Localization" NATO ASI Series B: Physical 308, 1993

[Ka] Kato, T: Perturbation Theory for Linear Operators Berlin-Heidelberg-New York: Spinger-Verlag, 1976

[KM1] Kitsch, W and Martinelli, F: On the Ergodic Properties of the Spectrum of General Random Opcrators J Reine Angew Math 334, 141-156 (1982)

[KM2] Kissch, W and Martinelli, F : On the Spectrum of Schrödinger Operators with a Random Potential Commun Math Phys 85, 329-350 (1982)

[Kp] Klopp, F : Localization for some continuous random Schroedinger operators Commun Math Phys 167, 553-569 (1995)

[Ku] Kuchment, P : Floquet Theory for Partial Differential Equations Basel: Birkhäuser Verlag, 1993

[M] Maynard, J : Acoustic Anderson Localization In: "Random Media and Composites," SIAM, 1988

[MI] Morse, P M and Ingard, K U : Theoretical Acoustics Princeton, NJ: Pinceton University Press, 1986

[PF] Pastu, L and Figotin, A : Spectra of Random and Almost-periodic Operators BerlinHeidelberg-New York: Springet-Verlag, 1991

[RS1] Reed, $M$ and Simon, B : Methods of Modern Mathematical Physics Vol I, Analysis of Operators New York: Academic Press, 1972

[RS3] Reed, M and Simon, B: Methods of Modern Mathematical Physics Vol III, Scattering Theory, New York: Academic Press, 1979

[RS4] Reed, M and Simon, B: Methods of Modern Mathematical Physics Vol IV, Analysis of Operators, New York: Academic Press, 1978

[Sc] Scattering and Localization of Classical Waves Ed by P Sheng, Singapore: World Scientific, 1990

[Sp] Spencer, T: Localization for Random and Quasiperiodic Potentials J Stat Phys 51, 1009-1019 (1988)

[VP] Villeneuve, P R and Piché, M: Photonic Band Gaps in Periodic Dielectric Structures Plog Quant Electr 18, 153-200 (1994) 